\title{
System Study: Auxiliary Feedwater 1998-2014
}

John A. Schroeder

December 2015

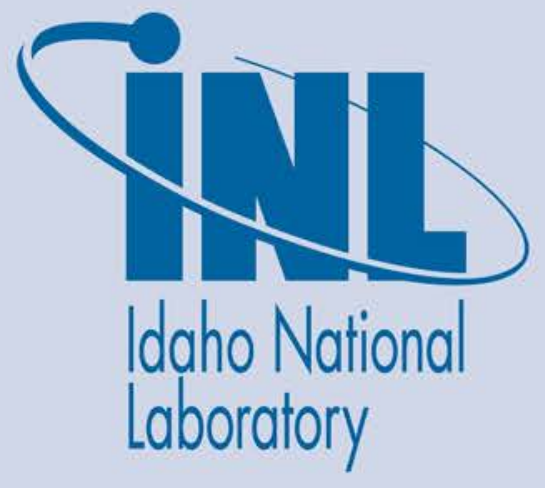

The INL is a U.S. Department of Energy National Laboratory operated by Battelle Energy Alliance 
NOTICE

This information was prepared as an account of work sponsored by an agency of the U.S. Government. Neither the U.S. Government nor any agency thereof, nor any of their employees, makes any warranty, express or implied, or assumes any legal liability or responsibility for any third party's use, or the results of such use, of any information, apparatus, product, or process disclosed herein, or represents that its use by such third party would not infringe privately owned rights. The views expressed herein are not necessarily those of the U.S. Nuclear Regulatory Commission. 


\title{
System Study: Auxiliary Feedwater 1998-2014
}

\author{
John A. Schroeder
}

Update Completed December 2015

Idaho National Laboratory

Risk Assessment and Management Services Department Idaho Falls, Idaho 83415

http://www.inl.gov

Prepared for the Division of Risk Assessment Office of Nuclear Regulatory Research

U.S. Nuclear Regulatory Commission NRC Agreement Number NRC-HQ-14-D-0018 



\begin{abstract}
This report presents an unreliability evaluation of the auxiliary feedwater (AFW) system at 69 U.S. commercial nuclear power plants. Demand, run hours, and failure data from fiscal year 1998 through 2014 for selected components were obtained from the Institute of Nuclear Power Operations (INPO)

Consolidated Events Database (ICES). The unreliability results are trended for the most recent 10-year period while yearly estimates for system unreliability are provided for the entire active period. No statistically significant increasing or decreasing trends were identified in the AFW results.
\end{abstract}


System Study

Auxiliary Feedwater
2014 Update

December 2015 


\section{CONTENTS}

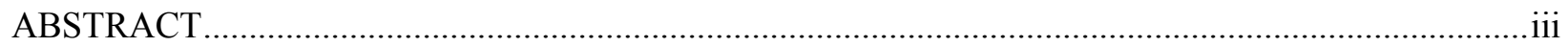

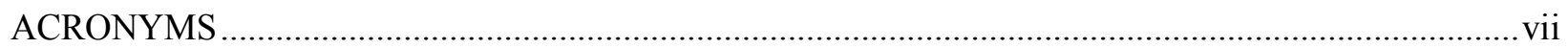

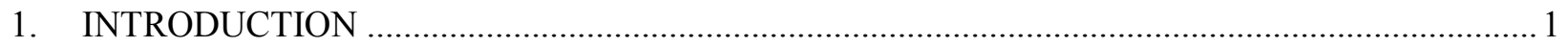

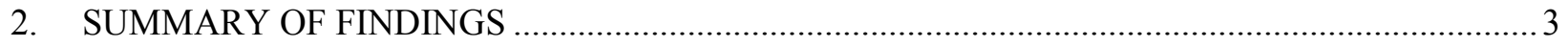

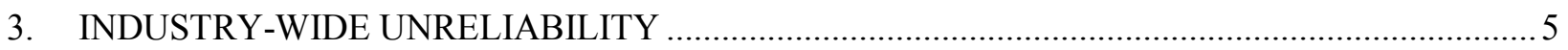

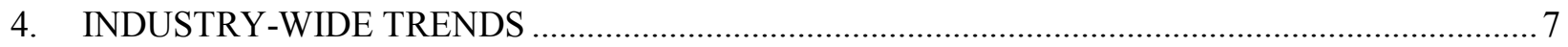

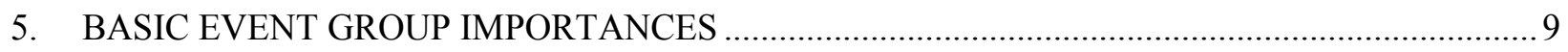

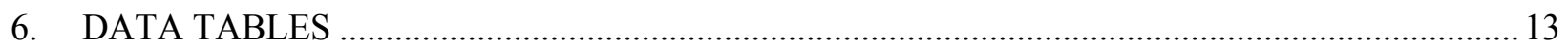

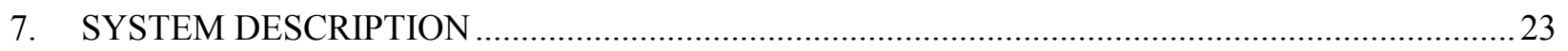

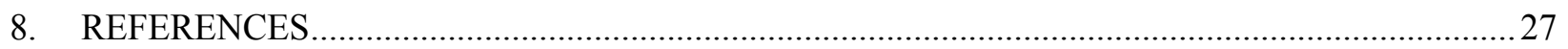

\section{FIGURES}

1. AFW start-only mission unreliability for Class 2,3 , and 4 and industry-wide groupings..................... 6

2. AFW 8-hour mission unreliability for Class 2,3 , and 4 and industry-wide groupings........................ 6

3. Trend of AFW system unreliability (start-only model), as a function of fiscal year. .......................... 8

4. Trend of AFW system unreliability (8-hour model), as a function of fiscal year................................ 8

5. AFW industry-wide basic event group importances. .................................................................. 9

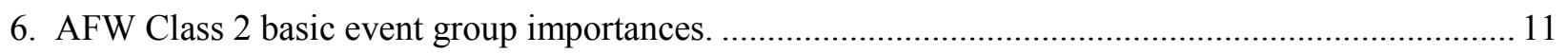

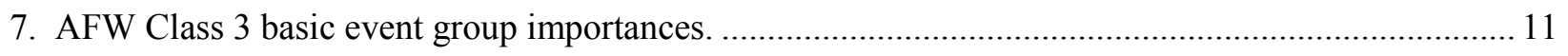

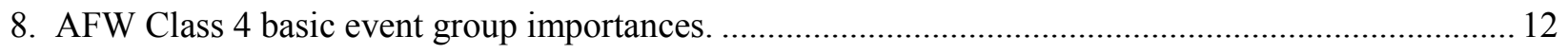




\section{TABLES}

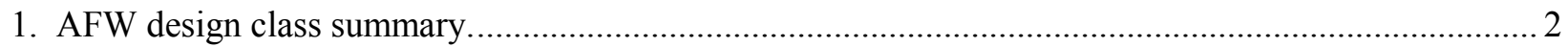

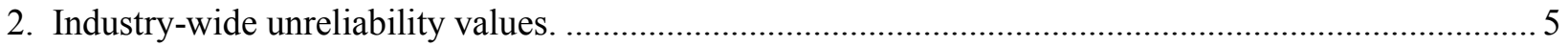

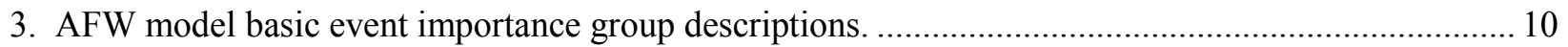

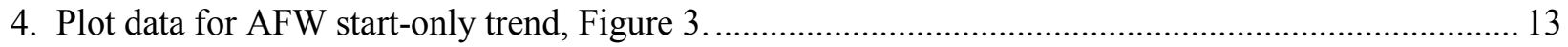

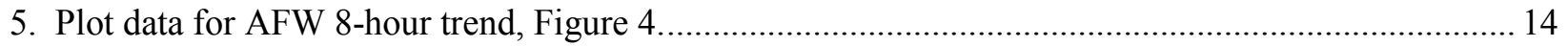

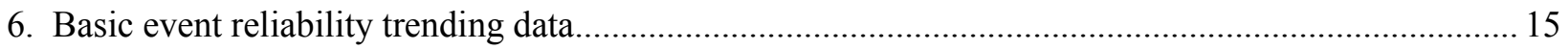

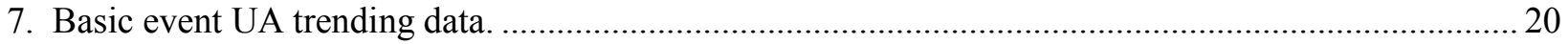

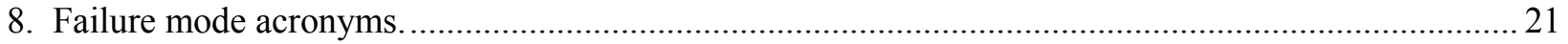

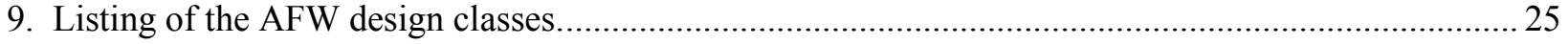




\title{
ACRONYMS
}

\author{
AFW auxiliary feedwater \\ AOV air-operated valve \\ $\mathrm{CCF} \quad$ common-cause failure \\ EPIX Equipment Performance and Information Exchange \\ ESFAS engineered safety features actuation system
}

FTLR fail to load/run

FTOC fail to open/close

FTOP fail to operate

FTR fail to run

FTR $<1 \mathrm{H}$ fail to run less than one hour (after start)

FTS fail to start

FY fiscal year

ICES INPO Consolidated Events Database

INPO Institute of Nuclear Power Operations

MDP motor-driven pump

MOV motor-operated valve

MSPI Mitigating Systems Performance Index

PRA probabilistic risk assessment

SO spurious operation

SPAR standardized plant analysis risk

SSU safety system unavailability

TDP turbine-driven pump

UA unavailability (maintenance or state of another component) 
System Study

Auxiliary Feedwater
2014 Update December 2015 


\section{System Study: Auxiliary Feedwater 1998-2014}

\section{INTRODUCTION}

This report presents an unreliability evaluation of the auxiliary feedwater (AFW) system at 69 U.S. commercial nuclear power plants listed in Table 1. For each plant, the corresponding Standardized Plant Analysis Risk (SPAR) model (version model indicated in Table 1) was used in the yearly calculations. Demand, run hours, and failure data from fiscal year (FY)-98 through FY-14 for selected components in the AFW were obtained from the Institute of Nuclear Power Operations (INPO) Consolidated Events Database (ICES). Train unavailability data (outages from test or maintenance) were obtained from the Reactor Oversight Process Safety System Unavailability (SSU) database (FY-98 through FY-01) and the Mitigating Systems Performance Index (MSPI) database (FY-02 through FY-14). Common-cause failure (CCF) data used in the models are from the 2010 update to the CCF database. The system unreliability results are trended for the most recent 10-year period while yearly estimates for system unreliability are provided for the entire active period.

This report does not attempt to estimate basic event values for use in a probabilistic risk assessment (PRA). Suggested values for such use are presented in the 2010 Component Reliability Update (Reference 1), which is an update to Reference 2 (NUREG/CR-6928). Baseline AFW unreliability results using basic event values from that report are summarized in Section 3. Trend results for AFW (using system-specific data) are presented in Section 4. Similar to previous system study updates, Section 5 contains importance information (using the baseline results from Section 3), and Section 7 describes the AFW.

The AFW classes were categorized by number of pump trains (no specification on pump type) used in the SPAR models. Class 2 AFW includes configurations that effectively result in a success criterion of one of two pumps. Class 3 AFW includes configurations that effectively result in a success criterion of one of three pumps. AFW designs effectively resulting in a success criterion of one of four or more are included in Class 4. Table 1 summarizes the plants and their classes.

The AFW model is evaluated using the transient flag set in the SPAR model. The transient flag set assumes all support systems are available and that the AFW system is required to perform to mitigate the effects of the transient initiating event. All models include failures due to unavailability while in test or maintenance. Human error has not been included in the SPAR model logic. An overview of the trending methods, glossary of terms, and abbreviations can be found in the Overview and Reference document on the Reactor Operational Experience Results and Databases web page.

Two modes of the models for the AFW system are calculated. The AFW start-only model is the SPAR AFW model modified by setting all fail-to-run basic events to zero (False), setting all recovery events to False, setting all pump-ends events to False, and setting all cooling basic events to False. The 8hour mission model includes all basic events in the SPAR AFW model. 
Table 1. AFW design class summary.

\begin{tabular}{|c|c|c|}
\hline Class & Plant & Version \\
\hline Class 2 & Arkansas 1 & 8.19 \\
\hline Class 2 & Braidwood 1 & 8.21 \\
\hline Class 2 & Braidwood 2 & 8.21 \\
\hline Class 2 & Byron 1 & 8.21 \\
\hline Class 2 & Byron 2 & 8.21 \\
\hline Class 2 & Crystal River 3 & 8.16 \\
\hline Class 2 & Prairie Island 1 & 8.19 \\
\hline Class 2 & Prairie Island 2 & 8.19 \\
\hline Class 2 & Seabrook & 8.20 \\
\hline Class 3 & Arkansas 2 & 8.21 \\
\hline Class 3 & Beaver Valley 2 & 8.23 \\
\hline Class 3 & Callaway & 8.21 \\
\hline Class 3 & Catawba 1 & 8.20 \\
\hline Class 3 & Catawba 2 & 8.20 \\
\hline Class 3 & Comanche Peak 1 & 8.21 \\
\hline Class 3 & Comanche Peak 2 & 8.21 \\
\hline Class 3 & Cook 1 & 8.20 \\
\hline Class 3 & Cook 2 & 8.20 \\
\hline Class 3 & Diablo Canyon 1 & 8.19 \\
\hline Class 3 & Diablo Canyon 2 & 8.19 \\
\hline Class 3 & Farley 1 & 8.18 \\
\hline Class 3 & Farley 2 & 8.18 \\
\hline Class 3 & Fort Calhoun & 8.20 \\
\hline Class 3 & Harris & 8.23 \\
\hline Class 3 & Indian Point 2 & 8.19 \\
\hline Class 3 & Indian Point 3 & 8.20 \\
\hline Class 3 & Kewaunee & 8.20 \\
\hline Class 3 & McGuire 1 & 8.20 \\
\hline Class 3 & McGuire 2 & 8.20 \\
\hline Class 3 & Millstone 2 & 8.17 \\
\hline Class 3 & Millstone 3 & 8.20 \\
\hline Class 3 & North Anna 1 & 8.20 \\
\hline Class 3 & North Anna 2 & 8.20 \\
\hline Class 3 & Oconee 1 & 8.19 \\
\hline Class 3 & Oconee 2 & 8.19 \\
\hline Class 3 & Oconee 3 & 8.19 \\
\hline Class 3 & Palisades & 8.20 \\
\hline
\end{tabular}




\section{SUMMARY OF FINDINGS}

The results of this AFW system unreliability study are summarized in this section. Of particular interest is the existence of any statistically significant ${ }^{\mathrm{a}}$ increasing trends. In this update, no statistically significant increasing trends were identified in the AFW unreliability trend results. In addition, this update identified no statistically significant decreasing trends in the AFW results.

The industry-wide AFW start-only and 8-hour basic event group importances were evaluated and are shown in Figure 5:

- In the Start-Only case - the leading contributor is the injection flow path followed by the TDP and MDP components (only the fail-to-start failure mode).

- In the 8-Hour case - the leading contributor to AFW system unreliability is the AFW motor-driven and turbine-driven pumps followed by recovery and the pump ends.

a. Statistically significant is defined in terms of the 'p-value.' A p-value is a probability indicating whether to accept or reject the null hypothesis that there is no trend in the data. P-values of less than or equal to 0.05 indicate that we are $95 \%$ confident that there is a trend in the data (reject the null hypothesis of no trend.) By convention, we use the "Michelin Guide" scale: p-value $<0.05$ (statistically significant), p-value $<0.01$ (highly statistically significant); pvalue $<0.001$ (extremely statistically significant). 
System Study

Auxiliary Feedwater
2014 Update

December 2015 


\section{INDUSTRY-WIDE UNRELIABILITY}

The AFW fault trees from the SPAR models were evaluated for each of the 69 operating U.S. commercial pressurized water nuclear power plants with an AFW system.

The industry-wide unreliability of the AFW system has been estimated for two modes of operation. A start-only model and an 8-hour mission model were evaluated. The uncertainty distributions for AFW show both plant design variability and parameter uncertainty while using industry-wide component failure data (FY 1998-FY 2010). ${ }^{a}$ Table 2 shows the percentiles and mean of the aggregated sample data (Latin hypercube, 1000 samples for each model) collected from the uncertainty calculations of the AFW fault trees in the SPAR models. In Figure 1 and Figure 2, the 5th and 95th percentiles and mean point estimates are shown for each class and for the industry.

In Figure 1 and Figure 2, the width of the distribution for a class is affected by the differences in the plant modeling and the parameter uncertainty used in the models. Because the width is affected by the plant modeling, the width is also affected by the number of different plant models in a class. For those classes with very few plants that share a design, the width can be very small.

Table 2. Industry-wide unreliability values.

\begin{tabular}{cccccc}
\hline Model & EPS Grouping & $\begin{array}{c}\text { Lower } \\
\mathbf{( 5 \% )}\end{array}$ & Median & Mean & $\begin{array}{c}\text { Upper } \\
\mathbf{( 9 5 \% )}\end{array}$ \\
\hline Start-only & Industry & $2.13 \mathrm{E}-08$ & $1.47 \mathrm{E}-06$ & $1.27 \mathrm{E}-05$ & $3.08 \mathrm{E}-05$ \\
& Class 2 & $3.66 \mathrm{E}-07$ & $9.10 \mathrm{E}-06$ & $2.60 \mathrm{E}-05$ & $1.07 \mathrm{E}-04$ \\
& Class 3 & $4.91 \mathrm{E}-08$ & $1.34 \mathrm{E}-06$ & $1.21 \mathrm{E}-05$ & $1.62 \mathrm{E}-05$ \\
& Class 4 & $7.06 \mathrm{E}-09$ & $5.22 \mathrm{E}-07$ & $2.46 \mathrm{E}-06$ & $1.08 \mathrm{E}-05$ \\
& Industry & $3.86 \mathrm{E}-07$ & $7.39 \mathrm{E}-06$ & $6.97 \mathrm{E}-05$ & $5.02 \mathrm{E}-04$ \\
8-hour Mission & Class 2 & $1.13 \mathrm{E}-06$ & $4.48 \mathrm{E}-05$ & $1.96 \mathrm{E}-04$ & $9.49 \mathrm{E}-04$ \\
& Class 3 & $8.47 \mathrm{E}-07$ & $7.35 \mathrm{E}-06$ & $5.70 \mathrm{E}-05$ & $5.03 \mathrm{E}-04$ \\
& Class 4 & $1.94 \mathrm{E}-08$ & $1.59 \mathrm{E}-06$ & $1.23 \mathrm{E}-05$ & $5.24 \mathrm{E}-05$ \\
\hline
\end{tabular}

a. By using industry-wide component failure data, individual plant performance is not included in the distribution of results. 


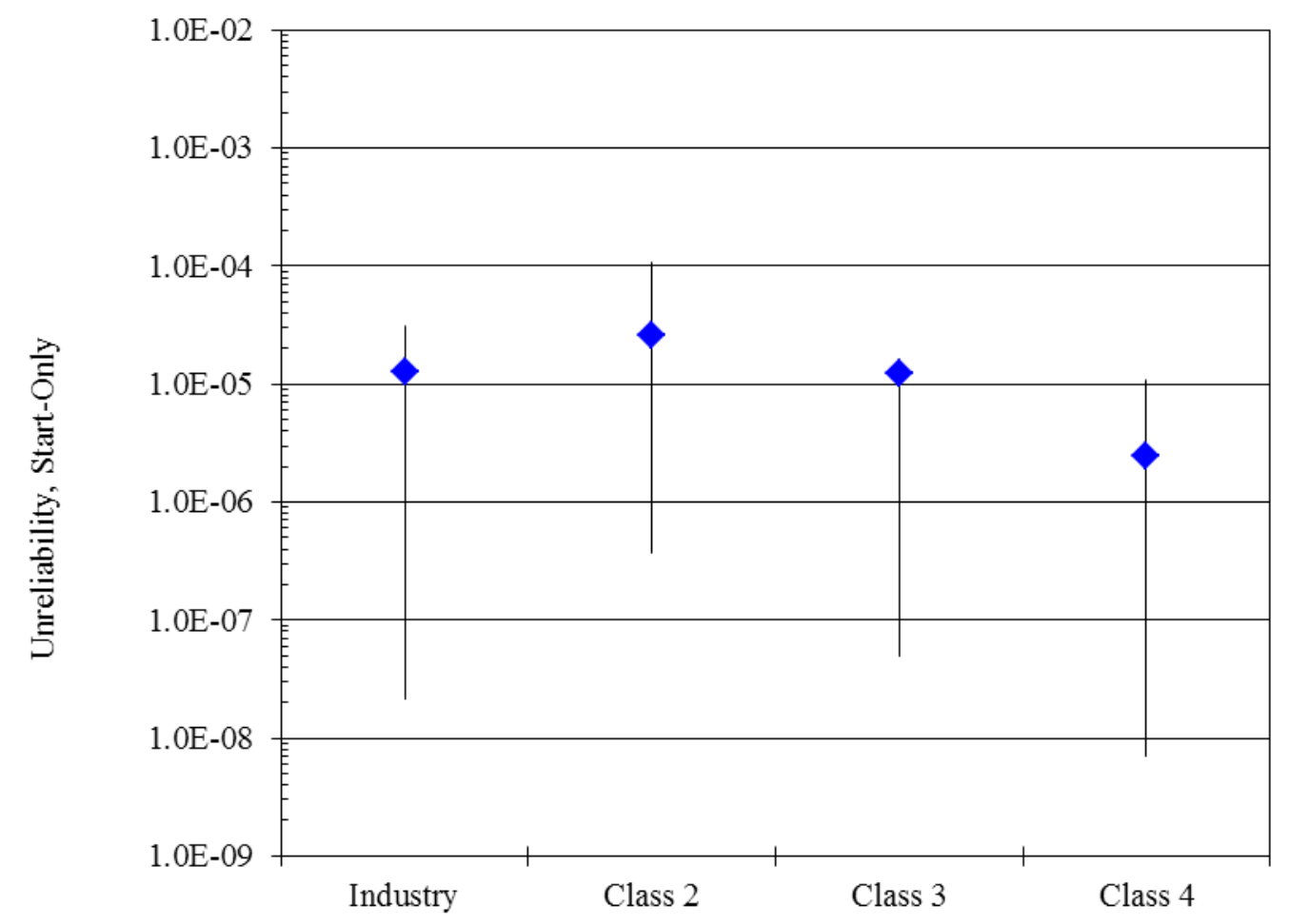

Figure 1. AFW start-only mission unreliability for Class 2, 3, and 4 and industry-wide groupings.

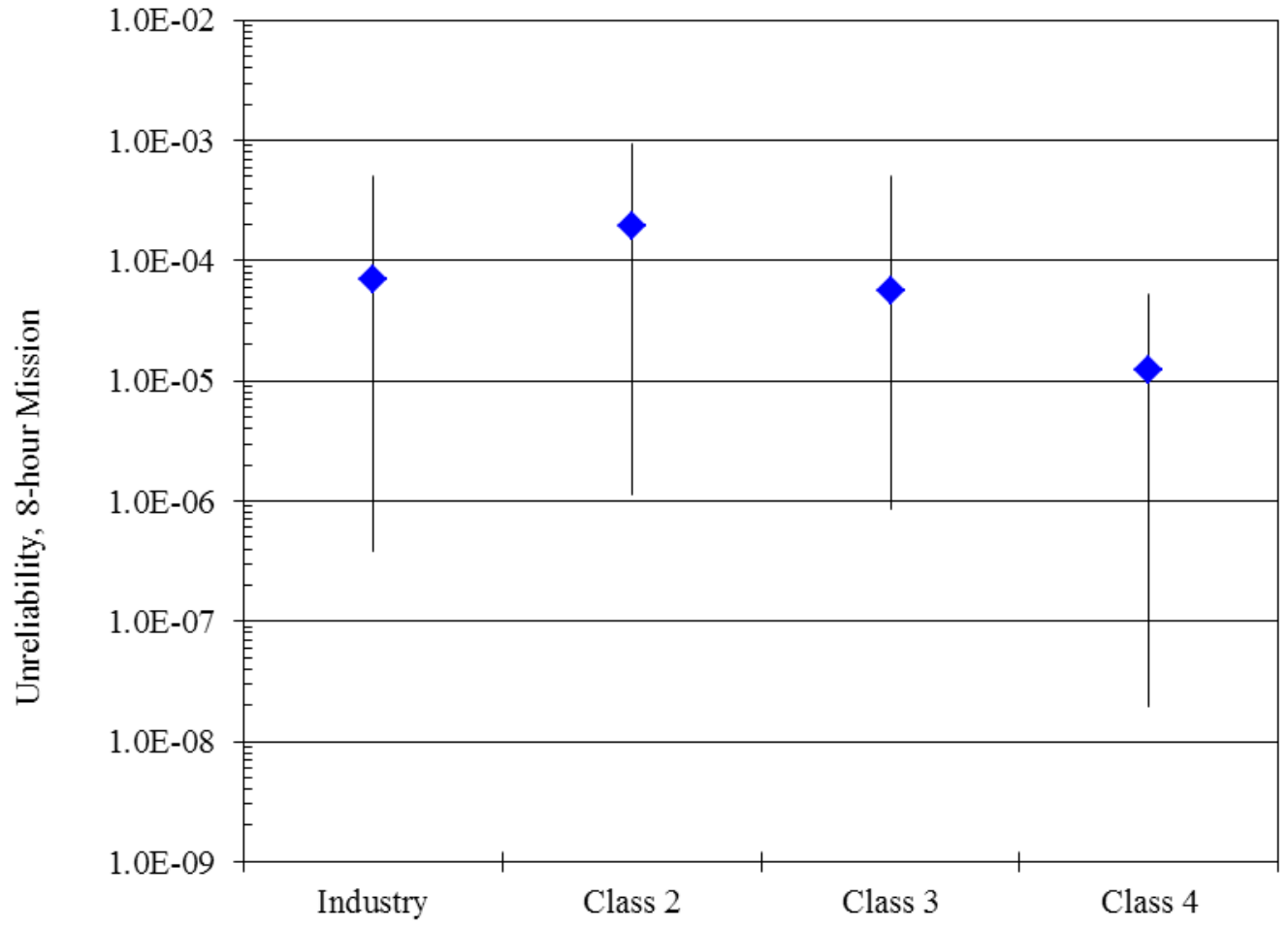

Figure 2. AFW 8-hour mission unreliability for Class 2, 3, and 4 and industry-wide groupings. 


\section{INDUSTRY-WIDE TRENDS}

The yearly (FY-98 through FY-14) failure and demand or run time data were obtained from ICES for the AFW system. AFW train maintenance unavailability data for trending are from the same time period, as reported in the ROP and ICES. The component basic event uncertainty was calculated for the AFW system components using the trending methods described in Section 1 and 2 of the Overview and Reference document. Tables 6 and 7 show the yearly data values for each AFW system specific component and failure mode combination that was varied in the model. These data were loaded into the AFW system fault tree in each SPAR model with an AFW system (see Table 1).

The trend charts show the results of varying component reliability data over time and updating generic, relatively flat prior distributions using data for each year. In addition, the calculated industrywide system reliability from this update (SPAR/ICES) is shown. Section 4 of the Overview and Reference link on the System Studies main web page provides more detailed discussion of the trending methods. In the lower left-hand corner of the trend figures, the regression method is reported.

The components that were varied in the AFW model are

- $\mathrm{AFW}$ motor-driven pump start, run, and test and maintenance.

- AFW turbine-driven pump start, run, and test and maintenance.

- Injection valves fail-to-open.

Figure 3 shows the trend in the AFW start-only model unreliability. Table 4 shows the data points for Figure 3. No statistically significant trends within the industry-wide estimates of AFW system start-only mission on a per fiscal year basis were identified. Figure 4 shows the trend in the 8-hour mission unreliability. No statistically significant trend within the industry-wide estimates of AFW system unreliability (8-hour mission) on a per fiscal year basis was identified. Table 5 shows the data points for Figure 4. 


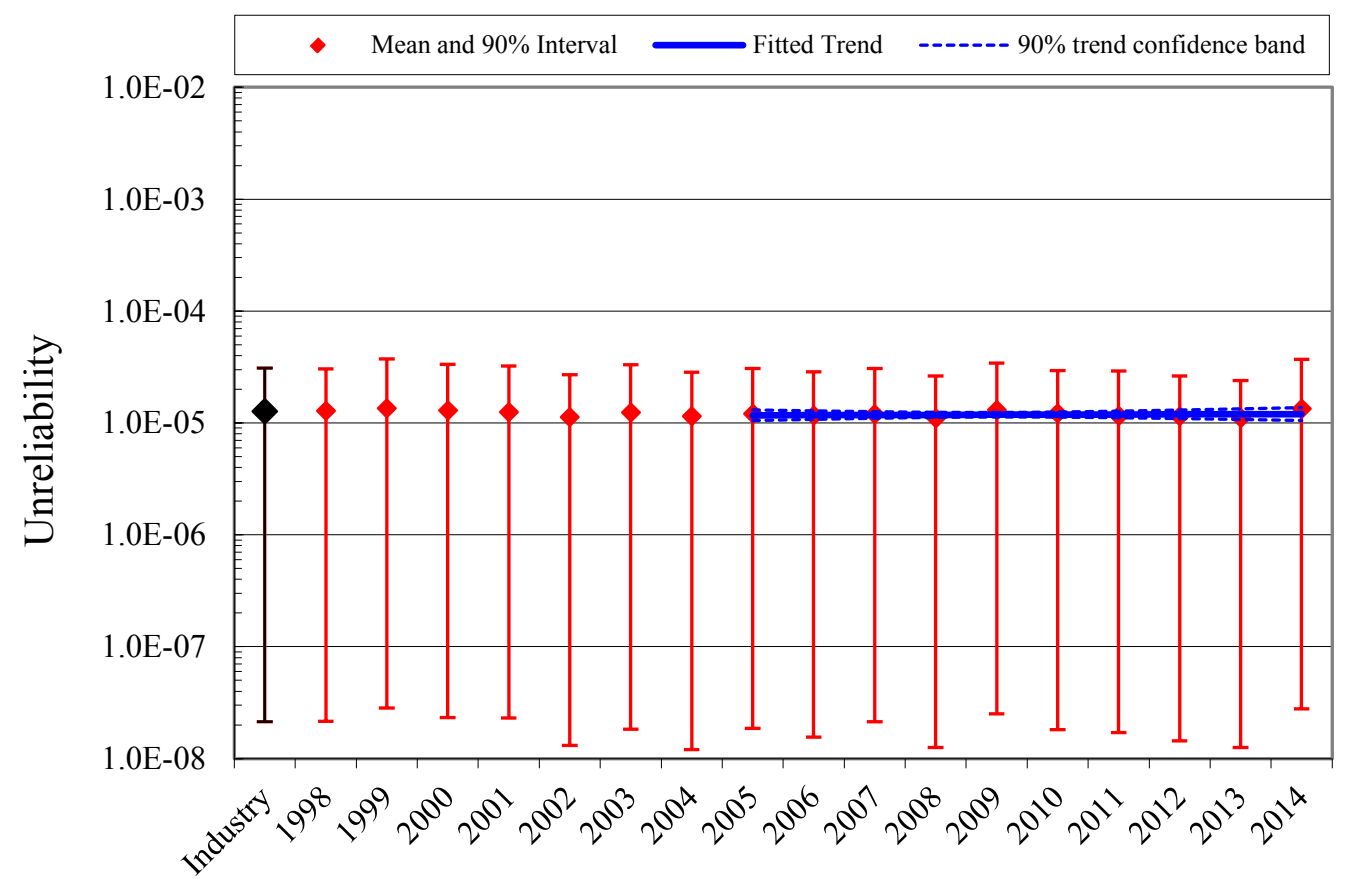

Logit model, $p$-value $=0.7218 \quad$ Fiscal Year

Industry AFW Start-Only 12-15-2015

Figure 3. Trend of AFW system unreliability (start-only model), as a function of fiscal year.

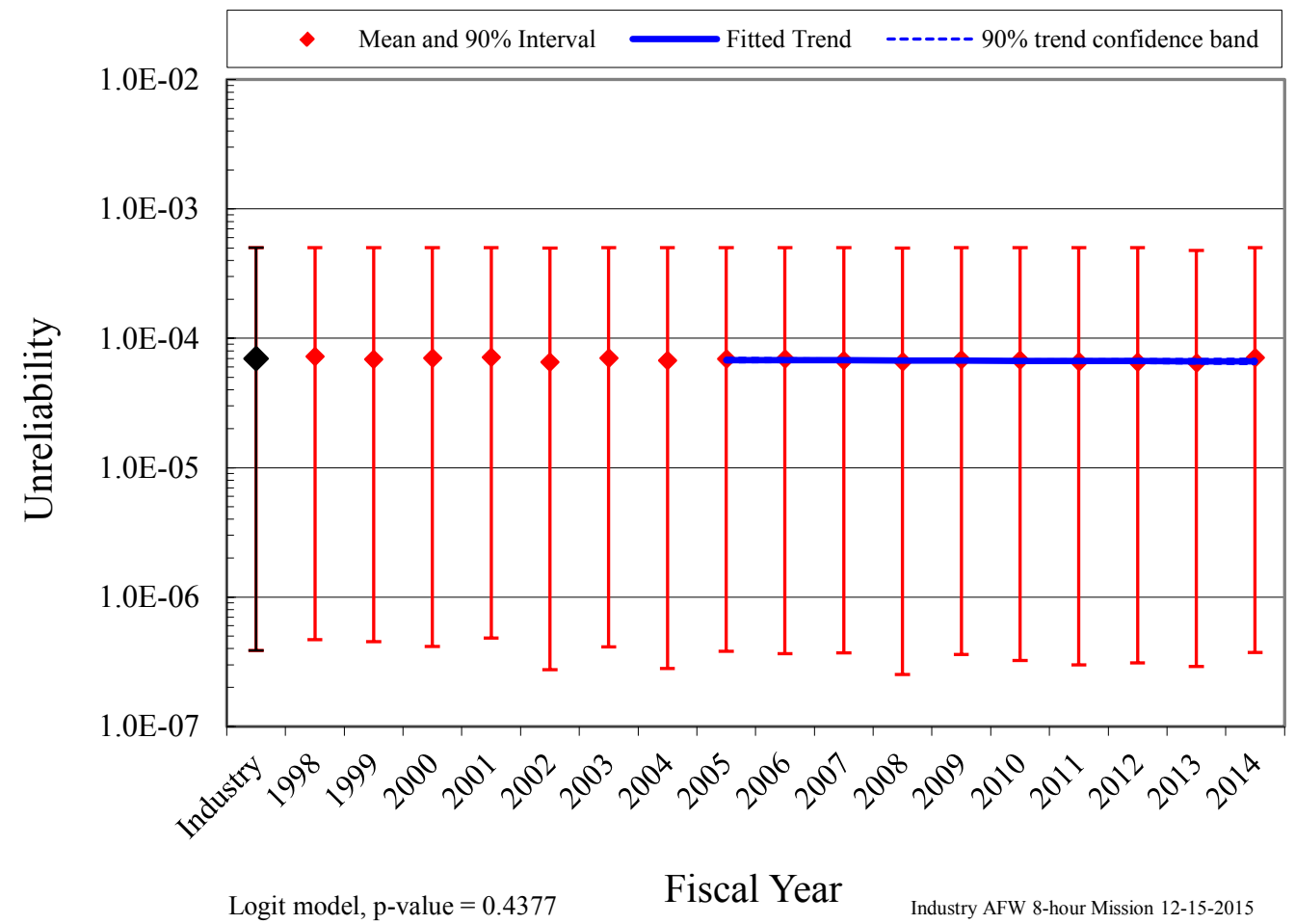

Figure 4. Trend of AFW system unreliability (8-hour model), as a function of fiscal year. 


\section{BASIC EVENT GROUP IMPORTANCES}

The AFW basic event group Fussell-Vesely importances were calculated for the start-only and 8-hour modes for each plant using the industry-wide data (1998-2010). These basic event group importances were then averaged across all plants to represent an industry-wide basic event group importance.

The industry-wide AFW start-only and 8-hour basic event group importances are shown in Figure 5:

- In the Start-Only case - the leading contributor is the injection flow path followed by the TDP and MDP components (only the fail-to-start failure mode).

- In the 8-Hour case - the leading contributor to AFW system unreliability is the AFW motor-driven and turbine-driven pumps followed by recovery and the pump ends.

For more discussion on the AFW motor/turbine-driven pumps, see the motor/turbine-driven pump component reliability studies at NRC Reactor Operational Experience Results and Databases. Table 3 shows the SPAR model AFW importance groups and their descriptions.

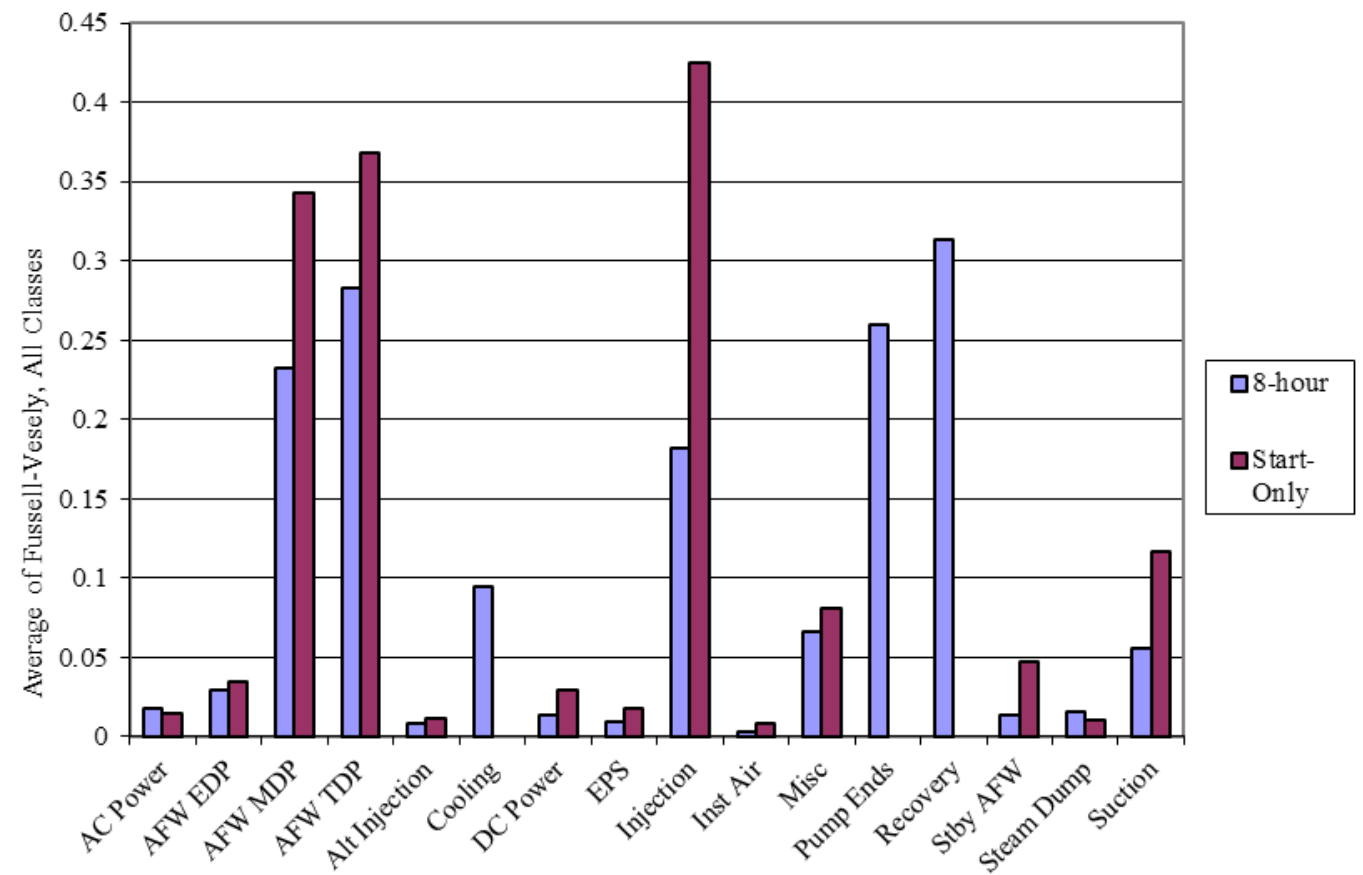

Basic Event Group

Figure 5. AFW industry-wide basic event group importances. 
Table 3. AFW model basic event importance group descriptions.

\begin{tabular}{|c|c|}
\hline Group & Description \\
\hline AC Power & The ac buses and circuit breakers that supply power to the AFW pumps. \\
\hline AFW EDP & $\begin{array}{l}\text { All basic events associated with the diesel engine-driven pumps. The start, run, } \\
\text { common-cause, and test and maintenance are included in this group of basic } \\
\text { events. }\end{array}$ \\
\hline AFW MDP & $\begin{array}{l}\text { All basic events associated with the motor-driven pumps. The start, run, } \\
\text { common-cause, and test and maintenance are included in this group of basic } \\
\text { events. }\end{array}$ \\
\hline AFW TDP & $\begin{array}{l}\text { All basic events associated with the turbine-driven pumps. The start, run, } \\
\text { common-cause, and test and maintenance are included in this group of basic } \\
\text { events. }\end{array}$ \\
\hline Alternate Injection & Alternate injection sources such as firewater. \\
\hline Cooling & $\begin{array}{l}\text { The pumps, valves, and heat exchangers that provide heat removal to the pumps. } \\
\text { In addition, the pumps, valves, air-conditioning equipment that are modeled to } \\
\text { provide room cooling to the AFW equipment }\end{array}$ \\
\hline DC Power & The batteries and battery chargers that supply power to the pump control circuitry. \\
\hline EPS & AFW dependency on the emergency power system. \\
\hline Injection & The motor-operated valves and check valves in the injection path. \\
\hline Inst Air & Instrument air support to the AFW model. \\
\hline Misc & Other events that are not typically modeled or of very low importance. \\
\hline Pump Ends & $\begin{array}{l}\text { The common-cause failure of the pump ends. Used to model common-cause } \\
\text { without the pump drivers. }\end{array}$ \\
\hline Recovery & $\begin{array}{l}\text { The operator recovery of the pump FTS, FTR, and other specialized modeled } \\
\text { recovery events. }\end{array}$ \\
\hline Special & $\begin{array}{l}\text { Various events used in the models that are not directly associated with the AFW } \\
\text { system. }\end{array}$ \\
\hline Suction & $\begin{array}{l}\text { The motor-operated valves and air-operated valves in the tank suction path. } \\
\text { Includes the failure of the tank. }\end{array}$ \\
\hline Stby AFW & $\begin{array}{l}\text { Standby means of injecting water to the steam generators. Includes startup } \\
\text { feedwater and cross-ties to adjacent units. }\end{array}$ \\
\hline
\end{tabular}

The basic event group importances were also averaged across plants of the same AFW class to represent class basic event group importances. The AFW class-specific start-only and 8-hour basic event group importances are shown in Figure 6 through Figure 8. 


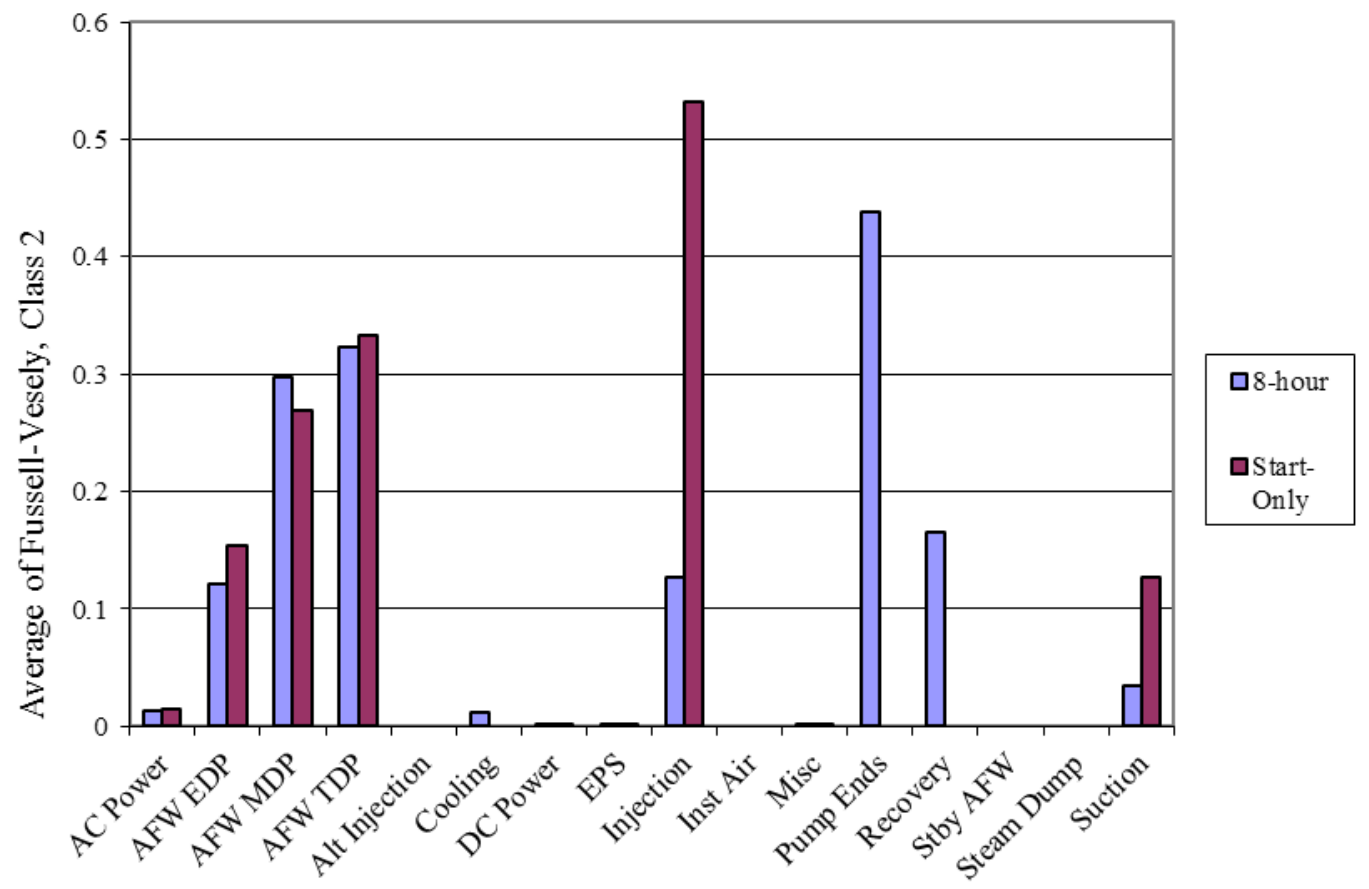

Basic Event Group

Figure 6. AFW Class 2 basic event group importances.

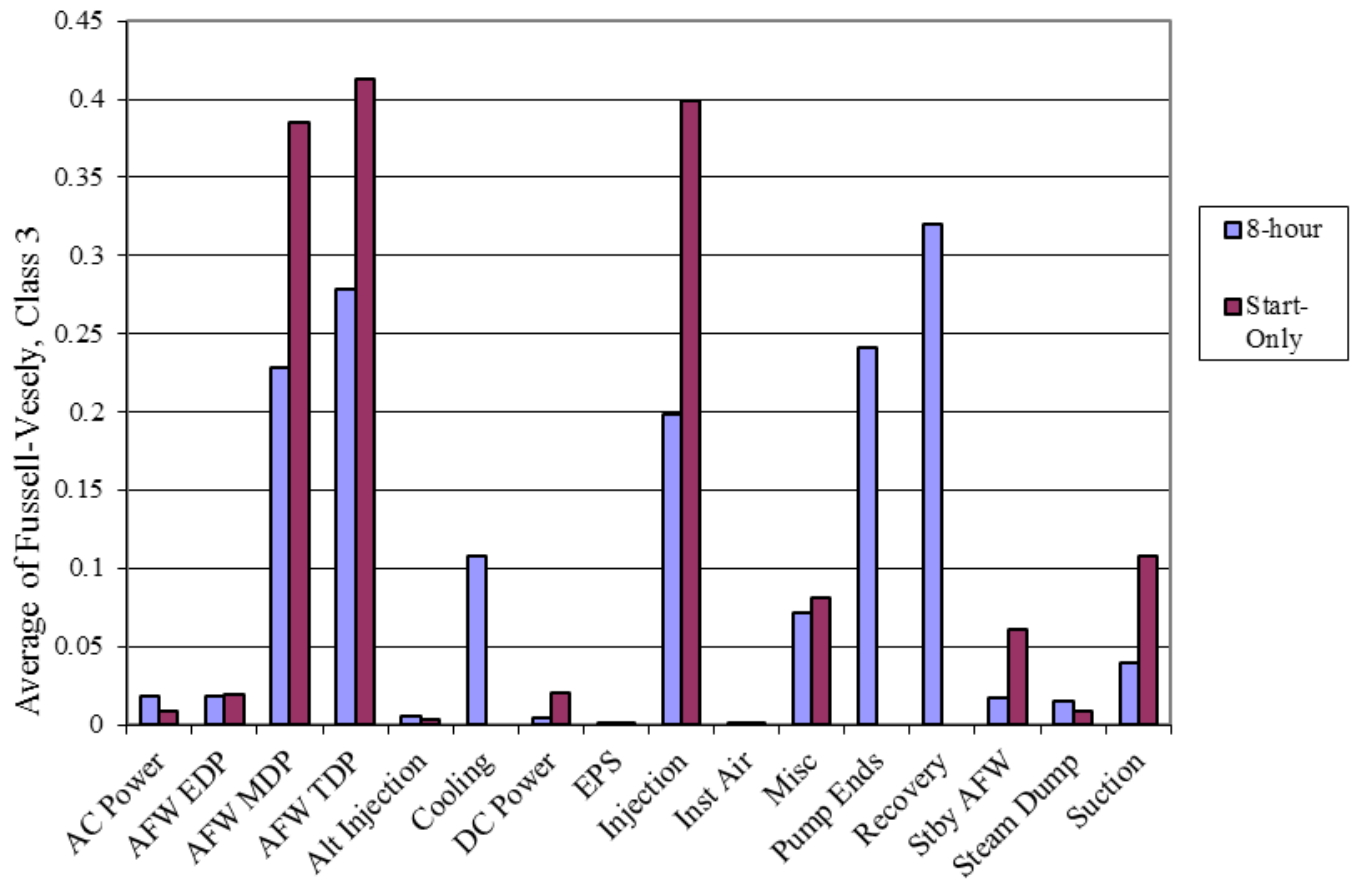

Basic Event Group

Figure 7. AFW Class 3 basic event group importances. 


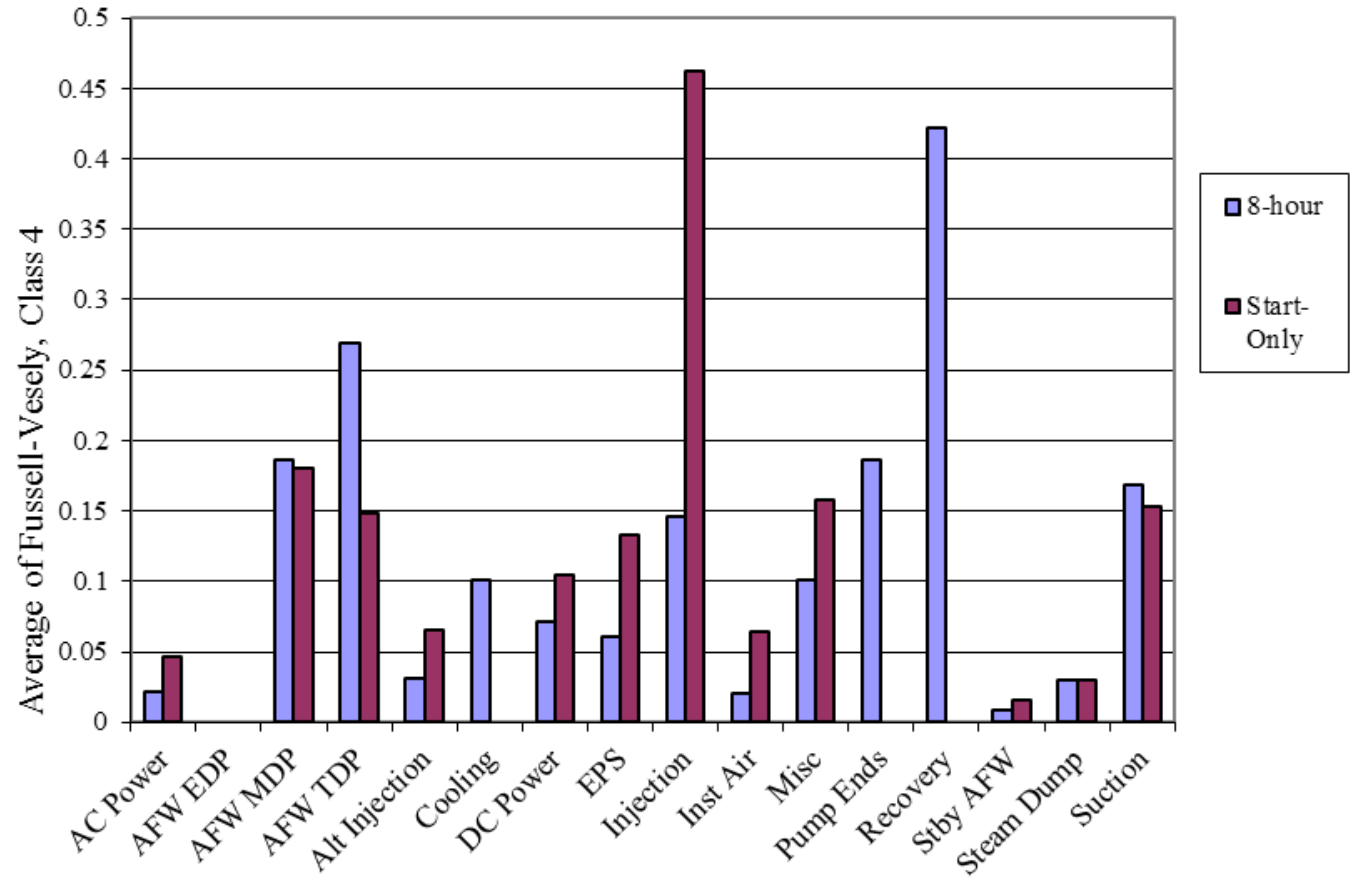

Basic Event Group

Figure 8. AFW Class 4 basic event group importances. 


\section{DATA TABLES}

Table 4. Plot data for AFW start-only trend, Figure 3.

\begin{tabular}{ccccccc}
\hline & \multicolumn{2}{c}{ Regression Curve Data Points } & \multicolumn{3}{c}{ Annual Estimate Data Points } \\
\cline { 2 - 7 } FY/Source & Mean & $\begin{array}{c}\text { Lower } \\
\mathbf{( 5 \% )}\end{array}$ & $\begin{array}{c}\text { Upper } \\
\mathbf{( 9 5 \% )}\end{array}$ & $\begin{array}{c}\text { Lower } \\
\mathbf{( 5 \% )}\end{array}$ & $\begin{array}{c}\text { Upper } \\
\mathbf{( 9 5 \% )}\end{array}$ & Mean \\
\hline SPAR/ICES & & & & $2.13 \mathrm{E}-08$ & $3.08 \mathrm{E}-05$ & $1.27 \mathrm{E}-05$ \\
1998 & & & & $2.16 \mathrm{E}-08$ & $3.05 \mathrm{E}-05$ & $1.27 \mathrm{E}-05$ \\
1999 & & & & $2.83 \mathrm{E}-08$ & $3.73 \mathrm{E}-05$ & $1.34 \mathrm{E}-05$ \\
\hline 2000 & & & & $2.32 \mathrm{E}-08$ & $3.35 \mathrm{E}-05$ & $1.29 \mathrm{E}-05$ \\
\hline 2001 & & & & $2.31 \mathrm{E}-08$ & $3.23 \mathrm{E}-05$ & $1.25 \mathrm{E}-05$ \\
2002 & & & & $1.31 \mathrm{E}-08$ & $2.70 \mathrm{E}-05$ & $1.12 \mathrm{E}-05$ \\
\hline 2003 & & & & $1.84 \mathrm{E}-08$ & $3.31 \mathrm{E}-05$ & $1.24 \mathrm{E}-05$ \\
\hline 2004 & & & & $1.20 \mathrm{E}-08$ & $2.83 \mathrm{E}-05$ & $1.14 \mathrm{E}-05$ \\
\hline 2005 & $1.17 \mathrm{E}-05$ & $1.05 \mathrm{E}-05$ & $1.31 \mathrm{E}-05$ & $1.87 \mathrm{E}-08$ & $3.05 \mathrm{E}-05$ & $1.20 \mathrm{E}-05$ \\
\hline 2006 & $1.17 \mathrm{E}-05$ & $1.08 \mathrm{E}-05$ & $1.28 \mathrm{E}-05$ & $1.56 \mathrm{E}-08$ & $2.85 \mathrm{E}-05$ & $1.16 \mathrm{E}-05$ \\
\hline 2007 & $1.18 \mathrm{E}-05$ & $1.10 \mathrm{E}-05$ & $1.26 \mathrm{E}-05$ & $2.14 \mathrm{E}-08$ & $3.08 \mathrm{E}-05$ & $1.19 \mathrm{E}-05$ \\
\hline 2008 & $1.18 \mathrm{E}-05$ & $1.12 \mathrm{E}-05$ & $1.25 \mathrm{E}-05$ & $1.25 \mathrm{E}-08$ & $2.64 \mathrm{E}-05$ & $1.09 \mathrm{E}-05$ \\
\hline 2009 & $1.18 \mathrm{E}-05$ & $1.13 \mathrm{E}-05$ & $1.24 \mathrm{E}-05$ & $2.50 \mathrm{E}-08$ & $3.42 \mathrm{E}-05$ & $1.30 \mathrm{E}-05$ \\
\hline 2010 & $1.19 \mathrm{E}-05$ & $1.13 \mathrm{E}-05$ & $1.25 \mathrm{E}-05$ & $1.81 \mathrm{E}-08$ & $2.94 \mathrm{E}-05$ & $1.22 \mathrm{E}-05$ \\
\hline 2011 & $1.19 \mathrm{E}-05$ & $1.11 \mathrm{E}-05$ & $1.27 \mathrm{E}-05$ & $1.70 \mathrm{E}-08$ & $2.92 \mathrm{E}-05$ & $1.15 \mathrm{E}-05$ \\
2012 & $1.19 \mathrm{E}-05$ & $1.09 \mathrm{E}-05$ & $1.30 \mathrm{E}-05$ & $1.44 \mathrm{E}-08$ & $2.62 \mathrm{E}-05$ & $1.14 \mathrm{E}-05$ \\
\hline 2013 & $1.20 \mathrm{E}-05$ & $1.07 \mathrm{E}-05$ & $1.34 \mathrm{E}-05$ & $1.26 \mathrm{E}-08$ & $2.39 \mathrm{E}-05$ & $1.10 \mathrm{E}-05$ \\
\hline 2014 & $1.20 \mathrm{E}-05$ & $1.05 \mathrm{E}-05$ & $1.37 \mathrm{E}-05$ & $2.79 \mathrm{E}-08$ & $3.70 \mathrm{E}-05$ & $1.34 \mathrm{E}-05$ \\
\hline
\end{tabular}


Table 5. Plot data for AFW 8-hour trend, Figure 4.

\begin{tabular}{ccccccc}
\hline & \multicolumn{2}{c}{ Regression Curve Data Points } & \multicolumn{3}{c}{ Annual Estimate Data Points } \\
\cline { 2 - 7 } FY/Source & Mean & $\begin{array}{c}\text { Lower } \\
\mathbf{( 5 \% )}\end{array}$ & $\begin{array}{c}\text { Upper } \\
\mathbf{( 9 5 \% )}\end{array}$ & $\begin{array}{c}\text { Lower } \\
\mathbf{( 5 \% )}\end{array}$ & $\begin{array}{c}\text { Upper } \\
\mathbf{( 9 5 \% )}\end{array}$ & Mean \\
\hline SPAR/ICES & & & & $3.86 \mathrm{E}-07$ & $5.02 \mathrm{E}-04$ & $6.97 \mathrm{E}-05$ \\
1998 & & & & $4.69 \mathrm{E}-07$ & $5.03 \mathrm{E}-04$ & $7.23 \mathrm{E}-05$ \\
\hline 1999 & & & & $4.51 \mathrm{E}-07$ & $5.02 \mathrm{E}-04$ & $6.86 \mathrm{E}-05$ \\
\hline 2000 & & & & $4.16 \mathrm{E}-07$ & $5.03 \mathrm{E}-04$ & $7.00 \mathrm{E}-05$ \\
\hline 2001 & & & & $4.81 \mathrm{E}-07$ & $5.03 \mathrm{E}-04$ & $7.10 \mathrm{E}-05$ \\
2002 & & & & $2.74 \mathrm{E}-07$ & $5.01 \mathrm{E}-04$ & $6.53 \mathrm{E}-05$ \\
\hline 2003 & & & & $4.11 \mathrm{E}-07$ & $5.03 \mathrm{E}-04$ & $7.00 \mathrm{E}-05$ \\
2004 & & & & $2.80 \mathrm{E}-07$ & $5.01 \mathrm{E}-04$ & $6.71 \mathrm{E}-05$ \\
\hline 2005 & $6.80 \mathrm{E}-05$ & $6.58 \mathrm{E}-05$ & $7.02 \mathrm{E}-05$ & $3.82 \mathrm{E}-07$ & $5.02 \mathrm{E}-04$ & $6.88 \mathrm{E}-05$ \\
\hline 2006 & $6.78 \mathrm{E}-05$ & $6.60 \mathrm{E}-05$ & $6.96 \mathrm{E}-05$ & $3.66 \mathrm{E}-07$ & $5.02 \mathrm{E}-04$ & $6.91 \mathrm{E}-05$ \\
\hline 2007 & $6.76 \mathrm{E}-05$ & $6.62 \mathrm{E}-05$ & $6.90 \mathrm{E}-05$ & $3.71 \mathrm{E}-07$ & $5.02 \mathrm{E}-04$ & $6.70 \mathrm{E}-05$ \\
\hline 2008 & $6.74 \mathrm{E}-05$ & $6.64 \mathrm{E}-05$ & $6.85 \mathrm{E}-05$ & $2.52 \mathrm{E}-07$ & $5.00 \mathrm{E}-04$ & $6.55 \mathrm{E}-05$ \\
\hline 2009 & $6.72 \mathrm{E}-05$ & $6.63 \mathrm{E}-05$ & $6.82 \mathrm{E}-05$ & $3.59 \mathrm{E}-07$ & $5.02 \mathrm{E}-04$ & $6.79 \mathrm{E}-05$ \\
\hline 2010 & $6.70 \mathrm{E}-05$ & $6.60 \mathrm{E}-05$ & $6.81 \mathrm{E}-05$ & $3.24 \mathrm{E}-07$ & $5.01 \mathrm{E}-04$ & $6.76 \mathrm{E}-05$ \\
\hline 2011 & $6.69 \mathrm{E}-05$ & $6.55 \mathrm{E}-05$ & $6.82 \mathrm{E}-05$ & $3.00 \mathrm{E}-07$ & $5.01 \mathrm{E}-04$ & $6.57 \mathrm{E}-05$ \\
\hline 2012 & $6.67 \mathrm{E}-05$ & $6.50 \mathrm{E}-05$ & $6.85 \mathrm{E}-05$ & $3.09 \mathrm{E}-07$ & $5.01 \mathrm{E}-04$ & $6.52 \mathrm{E}-05$ \\
\hline 2013 & $6.65 \mathrm{E}-05$ & $6.44 \mathrm{E}-05$ & $6.87 \mathrm{E}-05$ & $2.91 \mathrm{E}-07$ & $4.77 \mathrm{E}-04$ & $6.42 \mathrm{E}-05$ \\
\hline 2014 & $6.63 \mathrm{E}-05$ & $6.37 \mathrm{E}-05$ & $6.90 \mathrm{E}-05$ & $3.74 \mathrm{E}-07$ & $5.02 \mathrm{E}-04$ & $7.04 \mathrm{E}-05$ \\
\hline
\end{tabular}


Table 6. Basic event reliability trending data.

\begin{tabular}{|c|c|c|c|c|c|c|c|c|}
\hline \multirow{2}{*}{$\begin{array}{l}\text { Failure } \\
\text { Mode }\end{array}$} & \multirow[b]{2}{*}{ Component } & \multirow[b]{2}{*}{ Year } & \multirow{2}{*}{$\begin{array}{l}\text { Number of } \\
\text { Failures }\end{array}$} & \multirow{2}{*}{$\begin{array}{l}\text { Demands/ } \\
\text { Run Hours }\end{array}$} & \multicolumn{4}{|c|}{ Bayesian Update } \\
\hline & & & & & Mean & Post A & Post B & Distribution \\
\hline FTOC & AOV & 1998 & 5 & 1894.2 & 2.00E-03 & 6.112 & 3057.2 & Beta \\
\hline FTOC & AOV & 1999 & 0 & 2153.18 & 3.35E-04 & 1.112 & 3321.18 & Beta \\
\hline FTOC & AOV & 2000 & 2 & 1934.08 & 1.00E-03 & 3.112 & 3100.08 & Beta \\
\hline FTOC & AOV & 2001 & 3 & 2069.1 & 1.27E-03 & 4.112 & 3234.1 & Beta \\
\hline FTOC & AOV & 2002 & 3 & 2373 & 1.16E-03 & 4.112 & 3538 & Beta \\
\hline FTOC & AOV & 2003 & 0 & 2068.39 & 3.43E-04 & 1.112 & 3236.39 & Beta \\
\hline FTOC & AOV & 2004 & 0 & 2252.28 & 3.25E-04 & 1.112 & 3420.28 & Beta \\
\hline FTOC & AOV & 2005 & 2 & 2370.23 & 8.79E-04 & 3.112 & 3536.23 & Beta \\
\hline FTOC & AOV & 2006 & 2 & 1891.03 & $1.02 \mathrm{E}-03$ & 3.112 & 3057.03 & Beta \\
\hline FTOC & AOV & 2007 & 1 & 1871.68 & 6.95E-04 & 2.112 & 3038.68 & Beta \\
\hline FTOC & AOV & 2008 & 0 & 1756.84 & 3.80E-04 & 1.112 & 2924.84 & Beta \\
\hline FTOC & AOV & 2009 & 1 & 1724.76 & 7.30E-04 & 2.112 & 2891.76 & Beta \\
\hline FTOC & AOV & 2010 & 3 & 1754.28 & 1.41E-03 & 4.112 & 2919.28 & Beta \\
\hline FTOC & AOV & 2011 & 0 & 1761.44 & 3.79E-04 & 1.112 & 2929.44 & Beta \\
\hline FTOC & AOV & 2012 & 0 & 1808.28 & 3.73E-04 & 1.112 & 2976.28 & Beta \\
\hline FTOC & AOV & 2013 & 1 & 1821.56 & 7.06E-04 & 2.112 & 2988.56 & Beta \\
\hline FTOC & AOV & 2014 & 0 & 1709.01 & 3.86E-04 & 1.112 & 2877.01 & Beta \\
\hline FTOC & MOV & 1998 & 3 & 3600.36 & 8.81E-04 & 5.046 & 5720.36 & Beta \\
\hline FTOC & MOV & 1999 & 5 & 3692.68 & 1.21E-03 & 7.046 & 5810.68 & Beta \\
\hline FTOC & MOV & 2000 & 4 & 3909.47 & 1.00E-03 & 6.046 & 6028.47 & Beta \\
\hline FTOC & MOV & 2001 & 6 & 3709.62 & 1.38E-03 & 8.046 & 5826.62 & Beta \\
\hline FTOC & MOV & 2002 & 3 & 3944.27 & 8.31E-04 & 5.046 & 6064.27 & Beta \\
\hline FTOC & MOV & 2003 & 0 & 3865.28 & 3.42E-04 & 2.046 & 5988.28 & Beta \\
\hline FTOC & MOV & 2004 & 0 & 3966.71 & 3.36E-04 & 2.046 & 6089.71 & Beta \\
\hline FTOC & MOV & 2005 & 3 & 4072.61 & 8.14E-04 & 5.046 & 6192.61 & Beta \\
\hline FTOC & MOV & 2006 & 1 & 3698.37 & 5.23E-04 & 3.046 & 5820.37 & Beta \\
\hline FTOC & MOV & 2007 & 5 & 3659.23 & 1.22E-03 & 7.046 & 5777.23 & Beta \\
\hline FTOC & MOV & 2008 & 2 & 3739.31 & 6.90E-04 & 4.046 & 5860.31 & Beta \\
\hline FTOC & MOV & 2009 & 5 & 3708.34 & 1.21E-03 & 7.046 & 5826.34 & Beta \\
\hline FTOC & MOV & 2010 & 5 & 3712.61 & 1.21E-03 & 7.046 & 5830.61 & Beta \\
\hline FTOC & MOV & 2011 & 3 & 3629.37 & 8.77E-04 & 5.046 & 5749.37 & Beta \\
\hline FTOC & MOV & 2012 & 2 & 3455.59 & 7.25E-04 & 4.046 & 5576.59 & Beta \\
\hline FTOC & MOV & 2013 & 0 & 3457.1 & 3.67E-04 & 2.046 & 5580.1 & Beta \\
\hline FTOC & MOV & 2014 & 4 & 3475.67 & 1.08E-03 & 6.046 & 5594.67 & Beta \\
\hline FTOP & AOV & 1998 & 0 & 1699440 & $1.92 \mathrm{E}-07$ & 1.421 & 7418440 & Gamma \\
\hline FTOP & AOV & 1999 & 0 & 1699440 & 1.92E-07 & 1.421 & 7418440 & Gamma \\
\hline FTOP & AOV & 2000 & 1 & 1734480 & 3.25E-07 & 2.421 & 7453480 & Gamma \\
\hline FTOP & AOV & 2001 & 0 & 1734480 & 1.91E-07 & 1.421 & 7453480 & Gamma \\
\hline FTOP & AOV & 2002 & 0 & 1734480 & 1.91E-07 & 1.421 & 7453480 & Gamma \\
\hline FTOP & AOV & 2003 & 0 & 1734480 & 1.91E-07 & 1.421 & 7453480 & Gamma \\
\hline FTOP & AOV & 2004 & 0 & 1734480 & 1.91E-07 & 1.421 & 7453480 & Gamma \\
\hline
\end{tabular}


Table 6. (continued).

\begin{tabular}{|c|c|c|c|c|c|c|c|c|}
\hline \multirow{2}{*}{$\begin{array}{l}\text { Failure } \\
\text { Mode }\end{array}$} & \multirow[b]{2}{*}{ Component } & \multirow[b]{2}{*}{ Year } & \multirow{2}{*}{$\begin{array}{l}\text { Number of } \\
\text { Failures }\end{array}$} & \multirow{2}{*}{$\begin{array}{l}\text { Demands/ } \\
\text { Run Hours }\end{array}$} & \multicolumn{4}{|c|}{ Bayesian Update } \\
\hline & & & & & Mean & Post A & Post B & Distribution \\
\hline FTOP & AOV & 2005 & 0 & 1734480 & 1.91E-07 & 1.421 & 7453480 & Gamma \\
\hline FTOP & AOV & 2006 & 0 & 1734480 & 1.91E-07 & 1.421 & 7453480 & Gamma \\
\hline FTOP & AOV & 2007 & 0 & 1734480 & 1.91E-07 & 1.421 & 7453480 & Gamma \\
\hline FTOP & AOV & 2008 & 0 & 1734480 & $1.91 \mathrm{E}-07$ & 1.421 & 7453480 & Gamma \\
\hline FTOP & AOV & 2009 & 0 & 1734480 & 1.91E-07 & 1.421 & 7453480 & Gamma \\
\hline FTOP & AOV & 2010 & 1 & 1734480 & $3.25 \mathrm{E}-07$ & 2.421 & 7453480 & Gamma \\
\hline FTOP & AOV & 2011 & 0 & 1839600 & $1.88 \mathrm{E}-07$ & 1.421 & 7558600 & Gamma \\
\hline FTOP & AOV & 2012 & 0 & 1769520 & 1.90E-07 & 1.421 & $7.49 \mathrm{E}+06$ & Gamma \\
\hline FTOP & AOV & 2013 & 3 & 1769520 & 5.90E-07 & 4.421 & $7.49 \mathrm{E}+06$ & Gamma \\
\hline FTOP & AOV & 2014 & 1 & 1760760 & 3.24E-07 & 2.421 & $7.48 \mathrm{E}+06$ & Gamma \\
\hline FTOP & MOV & 1998 & 0 & 3942000 & 5.61E-08 & 1.458 & $2.60 \mathrm{E}+07$ & Gamma \\
\hline FTOP & MOV & 1999 & 0 & 3906960 & 5.62E-08 & 1.458 & $2.60 \mathrm{E}+07$ & Gamma \\
\hline FTOP & MOV & 2000 & 1 & 3906960 & $9.47 \mathrm{E}-08$ & 2.458 & $2.60 \mathrm{E}+07$ & Gamma \\
\hline FTOP & MOV & 2001 & 0 & 3906960 & 5.62E-08 & 1.458 & $2.60 \mathrm{E}+07$ & Gamma \\
\hline FTOP & MOV & 2002 & 1 & 3906960 & 9.47E-08 & 2.458 & $2.60 \mathrm{E}+07$ & Gamma \\
\hline FTOP & MOV & 2003 & 1 & 3906960 & $9.47 E-08$ & 2.458 & $2.60 \mathrm{E}+07$ & Gamma \\
\hline FTOP & MOV & 2004 & 2 & 3906960 & 1.33E-07 & 3.458 & $2.60 \mathrm{E}+07$ & Gamma \\
\hline FTOP & MOV & 2005 & 0 & 3906960 & 5.62E-08 & 1.458 & $2.60 \mathrm{E}+07$ & Gamma \\
\hline FTOP & MOV & 2006 & 0 & 3906960 & 5.62E-08 & 1.458 & $2.60 \mathrm{E}+07$ & Gamma \\
\hline FTOP & MOV & 2007 & 0 & 3906960 & 5.62E-08 & 1.458 & $2.60 \mathrm{E}+07$ & Gamma \\
\hline FTOP & MOV & 2008 & 1 & 3924480 & 9.46E-08 & 2.458 & $2.60 \mathrm{E}+07$ & Gamma \\
\hline FTOP & MOV & 2009 & 0 & 3994560 & 5.60E-08 & 1.458 & $2.60 \mathrm{E}+07$ & Gamma \\
\hline FTOP & MOV & 2010 & 1 & 3924480 & 9.46E-08 & 2.458 & $2.60 \mathrm{E}+07$ & Gamma \\
\hline FTOP & MOV & 2011 & 0 & 4047120 & 5.59E-08 & 1.458 & $2.61 \mathrm{E}+07$ & Gamma \\
\hline FTOP & MOV & 2012 & 1 & 3810600 & 9.50E-08 & 2.458 & $2.59 \mathrm{E}+07$ & Gamma \\
\hline FTOP & MOV & 2013 & 1 & 3828120 & $9.50 \mathrm{E}-08$ & 2.458 & $2.59 \mathrm{E}+07$ & Gamma \\
\hline FTOP & MOV & 2014 & 0 & 3828120 & 5.63E-08 & 1.458 & $2.59 \mathrm{E}+07$ & Gamma \\
\hline SO & AOV & 1998 & 0 & 1699440 & 9.84E-08 & 0.6801 & 6910440 & Gamma \\
\hline SO & AOV & 1999 & 0 & 1699440 & 9.84E-08 & 0.6801 & 6910440 & Gamma \\
\hline SO & AOV & 2000 & 0 & 1734480 & 9.79E-08 & 0.6801 & 6945480 & Gamma \\
\hline SO & AOV & 2001 & 1 & 1734480 & 2.42E-07 & 1.6801 & 6945480 & Gamma \\
\hline SO & AOV & 2002 & 0 & 1734480 & 9.79E-08 & 0.6801 & 6945480 & Gamma \\
\hline SO & AOV & 2003 & 1 & 1734480 & 2.42E-07 & 1.6801 & 6945480 & Gamma \\
\hline SO & AOV & 2004 & 1 & 1734480 & 2.42E-07 & 1.6801 & 6945480 & Gamma \\
\hline SO & AOV & 2005 & 1 & 1734480 & 2.42E-07 & 1.6801 & 6945480 & Gamma \\
\hline SO & AOV & 2006 & 0 & 1734480 & 9.79E-08 & 0.6801 & 6945480 & Gamma \\
\hline SO & AOV & 2007 & 1 & 1734480 & 2.42E-07 & 1.6801 & 6945480 & Gamma \\
\hline SO & AOV & 2008 & 1 & 1734480 & 2.42E-07 & 1.6801 & 6945480 & Gamma \\
\hline SO & AOV & 2009 & 1 & 1734480 & 2.42E-07 & 1.6801 & 6945480 & Gamma \\
\hline SO & AOV & 2010 & 0 & 1734480 & 9.79E-08 & 0.6801 & 6945480 & Gamma \\
\hline SO & AOV & 2011 & 1 & 1839600 & 2.38E-07 & 1.6801 & 7050600 & Gamma \\
\hline
\end{tabular}


Table 6. (continued).

\begin{tabular}{|c|c|c|c|c|c|c|c|c|}
\hline \multirow{2}{*}{$\begin{array}{c}\text { Failure } \\
\text { Mode }\end{array}$} & \multirow[b]{2}{*}{ Component } & \multirow[b]{2}{*}{ Year } & \multirow{2}{*}{$\begin{array}{l}\text { Number of } \\
\text { Failures }\end{array}$} & \multirow{2}{*}{$\begin{array}{l}\text { Demands/ } \\
\text { Run Hours }\end{array}$} & \multicolumn{4}{|c|}{ Bayesian Update } \\
\hline & & & & & Mean & Post A & Post B & Distribution \\
\hline $\mathrm{SO}$ & AOV & 2012 & 0 & 1769520 & $9.74 \mathrm{E}-08$ & 0.6801 & 6980520 & Gamma \\
\hline so & AOV & 2013 & 0 & 1769520 & $9.74 \mathrm{E}-08$ & 0.6801 & 6980520 & Gamma \\
\hline so & AOV & 2014 & 0 & 1760760 & 9.76E-08 & 0.6801 & 6971760 & Gamma \\
\hline So & MOV & 1998 & 1 & 3942000 & 7.56E-08 & 1.5703 & $2.08 E+07$ & Gamma \\
\hline SO & MOV & 1999 & 0 & 3906960 & $2.75 \mathrm{E}-08$ & 0.5703 & $2.07 E+07$ & Gamma \\
\hline so & MOV & 2000 & 1 & 3906960 & 7.57E-08 & 1.5703 & $2.07 E+07$ & Gamma \\
\hline SO & MOV & 2001 & 1 & 3906960 & 7.57E-08 & 1.5703 & $2.07 E+07$ & Gamma \\
\hline So & MOV & 2002 & 0 & 3906960 & 2.75E-08 & 0.5703 & $2.07 E+07$ & Gamma \\
\hline SO & MOV & 2003 & 0 & 3906960 & $2.75 \mathrm{E}-08$ & 0.5703 & $2.07 E+07$ & Gamma \\
\hline So & MOV & 2004 & 0 & 3906960 & 2.75E-08 & 0.5703 & $2.07 E+07$ & Gamma \\
\hline SO & MOV & 2005 & 0 & 3906960 & 2.75E-08 & 0.5703 & $2.07 E+07$ & Gamma \\
\hline so & MOV & 2006 & 0 & 3906960 & 2.75E-08 & 0.5703 & $2.07 E+07$ & Gamma \\
\hline So & MOV & 2007 & 0 & 3906960 & 2.75E-08 & 0.5703 & $2.07 E+07$ & Gamma \\
\hline so & MOV & 2008 & 0 & 3924480 & $2.75 \mathrm{E}-08$ & 0.5703 & $2.08 E+07$ & Gamma \\
\hline So & MOV & 2009 & 0 & 3994560 & 2.74E-08 & 0.5703 & $2.08 E+07$ & Gamma \\
\hline so & MOV & 2010 & 0 & 3924480 & $2.75 \mathrm{E}-08$ & 0.5703 & $2.08 E+07$ & Gamma \\
\hline So & MOV & 2011 & 0 & 4047120 & 2.73E-08 & 0.5703 & $2.09 E+07$ & Gamma \\
\hline so & MOV & 2012 & 0 & 3810600 & 2.76E-08 & 0.5703 & $2.07 E+07$ & Gamma \\
\hline So & MOV & 2013 & 0 & 3828120 & 2.76E-08 & 0.5703 & $2.07 E+07$ & Gamma \\
\hline SO & MOV & 2014 & 0 & 3828120 & 2.76E-08 & 0.5703 & $2.07 E+07$ & Gamma \\
\hline $\mathrm{FTR}<1 \mathrm{H}$ & MDP & 1998 & 1 & 1685.12 & 1.71E-04 & 2.82 & $1.65 E+04$ & Gamma \\
\hline $\mathrm{FTR}<1 \mathrm{H}$ & MDP & 1999 & 2 & 1636.93 & 2.33E-04 & 3.82 & $1.64 E+04$ & Gamma \\
\hline $\mathrm{FTR}<1 \mathrm{H}$ & MDP & 2000 & 1 & 1726.26 & 1.71E-04 & 2.82 & $1.65 E+04$ & Gamma \\
\hline $\mathrm{FTR}<1 \mathrm{H}$ & MDP & 2001 & 0 & 1807.29 & 1.10E-04 & 1.82 & $1.66 \mathrm{E}+04$ & Gamma \\
\hline $\mathrm{FTR}<1 \mathrm{H}$ & MDP & 2002 & 2 & 1893.23 & 2.29E-04 & 3.82 & $1.67 E+04$ & Gamma \\
\hline $\mathrm{FTR}<1 \mathrm{H}$ & MDP & 2003 & 0 & 2035.1 & $1.08 \mathrm{E}-04$ & 1.82 & $1.68 \mathrm{E}+04$ & Gamma \\
\hline $\mathrm{FTR}<1 \mathrm{H}$ & MDP & 2004 & 1 & 2130.03 & 1.67E-04 & 2.82 & $1.69 E+04$ & Gamma \\
\hline $\mathrm{FTR}<1 \mathrm{H}$ & MDP & 2005 & 2 & 2204.32 & $2.25 \mathrm{E}-04$ & 3.82 & $1.70 \mathrm{E}+04$ & Gamma \\
\hline $\mathrm{FTR}<1 \mathrm{H}$ & MDP & 2006 & 0 & 1938.64 & 1.09E-04 & 1.82 & $1.67 E+04$ & Gamma \\
\hline $\mathrm{FTR}<1 \mathrm{H}$ & MDP & 2007 & 1 & 2220.11 & 1.66E-04 & 2.82 & $1.70 E+04$ & Gamma \\
\hline $\mathrm{FTR}<1 \mathrm{H}$ & MDP & 2008 & 0 & 2007.37 & 1.08E-04 & 1.82 & $1.68 E+04$ & Gamma \\
\hline $\mathrm{FTR}<1 \mathrm{H}$ & MDP & 2009 & 0 & 1841.06 & 1.09E-04 & 1.82 & $1.66 \mathrm{E}+04$ & Gamma \\
\hline $\mathrm{FTR}<1 \mathrm{H}$ & MDP & 2010 & 1 & 2078.23 & 1.67E-04 & 2.82 & $1.69 E+04$ & Gamma \\
\hline $\mathrm{FTR}<1 \mathrm{H}$ & MDP & 2011 & 0 & 2050.25 & 1.08E-04 & 1.82 & $1.68 E+04$ & Gamma \\
\hline $\mathrm{FTR}<1 \mathrm{H}$ & MDP & 2012 & 0 & 1860.84 & 1.09E-04 & 1.82 & $1.67 E+04$ & Gamma \\
\hline $\mathrm{FTR}<1 \mathrm{H}$ & MDP & 2013 & 2 & 2055.01 & 2.27E-04 & 3.82 & $1.68 E+04$ & Gamma \\
\hline $\mathrm{FTR}<1 \mathrm{H}$ & MDP & 2014 & 0 & 1799.73 & 1.10E-04 & 1.82 & $1.66 \mathrm{E}+04$ & Gamma \\
\hline $\mathrm{FTR}<1 \mathrm{H}$ & TDP & 1998 & 2 & 1065.11 & 2.31E-03 & 2.9618 & $1.28 E+03$ & Gamma \\
\hline $\mathrm{FTR}<1 \mathrm{H}$ & TDP & 1999 & 3 & 976.19 & 3.32E-03 & 3.9618 & $1.19 E+03$ & Gamma \\
\hline $\mathrm{FTR}<1 \mathrm{H}$ & TDP & 2000 & 2 & 979.61 & 2.48E-03 & 2.9618 & $1.20 E+03$ & Gamma \\
\hline $\mathrm{FTR}<1 \mathrm{H}$ & TDP & 2001 & 4 & 913.85 & 4.39E-03 & 4.9618 & $1.13 E+03$ & Gamma \\
\hline
\end{tabular}


Table 6. (continued).

\begin{tabular}{|c|c|c|c|c|c|c|c|c|}
\hline \multirow{2}{*}{$\begin{array}{l}\text { Failure } \\
\text { Mode }\end{array}$} & \multirow[b]{2}{*}{ Component } & \multirow[b]{2}{*}{ Year } & \multirow{2}{*}{$\begin{array}{l}\text { Number of } \\
\text { Failures }\end{array}$} & \multirow{2}{*}{$\begin{array}{l}\text { Demands/ } \\
\text { Run Hours }\end{array}$} & \multicolumn{4}{|c|}{ Bayesian Update } \\
\hline & & & & & Mean & Post A & Post B & Distribution \\
\hline $\mathrm{FTR}<1 \mathrm{H}$ & TDP & 2002 & 2 & 911.77 & 2.63E-03 & 2.9618 & $1.13 E+03$ & Gamma \\
\hline $\mathrm{FTR}<1 \mathrm{H}$ & TDP & 2003 & 7 & 974.78 & 6.68E-03 & 7.9618 & 1.19E+03 & Gamma \\
\hline $\mathrm{FTR}<1 \mathrm{H}$ & TDP & 2004 & 3 & 994.49 & 3.27E-03 & 3.9618 & $1.21 \mathrm{E}+03$ & Gamma \\
\hline $\mathrm{FTR}<1 \mathrm{H}$ & TDP & 2005 & 1 & 994.88 & $1.62 \mathrm{E}-03$ & 1.9618 & $1.21 \mathrm{E}+03$ & Gamma \\
\hline $\mathrm{FTR}<1 \mathrm{H}$ & TDP & 2006 & 1 & 986.51 & 1.63E-03 & 1.9618 & $1.20 \mathrm{E}+03$ & Gamma \\
\hline $\mathrm{FTR}<1 \mathrm{H}$ & TDP & 2007 & 2 & 971.91 & 2.49E-03 & 2.9618 & 1188.31 & Gamma \\
\hline FTR $<1 \mathrm{H}$ & TDP & 2008 & 3 & 984.15 & 3.30E-03 & 3.9618 & 1200.55 & Gamma \\
\hline FTR<1H & TDP & 2009 & 4 & 1089.29 & $3.80 \mathrm{E}-03$ & 4.9618 & 1305.69 & Gamma \\
\hline FTR $<1 \mathrm{H}$ & TDP & 2010 & 2 & 1147.46 & 2.17E-03 & 2.9618 & 1363.86 & Gamma \\
\hline $\mathrm{FTR}<1 \mathrm{H}$ & TDP & 2011 & 1 & 1122.68 & $1.47 \mathrm{E}-03$ & 1.9618 & 1339.08 & Gamma \\
\hline $\mathrm{FTR}<1 \mathrm{H}$ & TDP & 2012 & 2 & 1047.37 & 2.34E-03 & 2.9618 & 1263.77 & Gamma \\
\hline FTR<1H & TDP & 2013 & 0 & 1129.13 & 7.15E-04 & 0.9618 & 1345.53 & Gamma \\
\hline $\mathrm{FTR}<1 \mathrm{H}$ & TDP & 2014 & 0 & 1056.95 & 7.55E-04 & 0.9618 & 1273.35 & Gamma \\
\hline $\mathrm{FTR}>1 \mathrm{H}$ & MDP & 1998 & 1 & 5836.18 & 2.20E-05 & 1.781 & 80846.18 & Gamma \\
\hline $\mathrm{FTR}>1 \mathrm{H}$ & MDP & 1999 & 0 & 9198.12 & $9.27 \mathrm{E}-06$ & 0.781 & 84208.12 & Gamma \\
\hline FTR $>1 \mathrm{H}$ & MDP & 2000 & 0 & 6576.42 & 9.57E-06 & 0.781 & 81586.42 & Gamma \\
\hline $\mathrm{FTR}>1 \mathrm{H}$ & MDP & 2001 & 5 & 9611.46 & 6.83E-05 & 5.781 & 84621.46 & Gamma \\
\hline $\mathrm{FTR}>1 \mathrm{H}$ & MDP & 2002 & 0 & 7900.25 & $9.42 E-06$ & 0.781 & 82910.25 & Gamma \\
\hline $\mathrm{FTR}>1 \mathrm{H}$ & MDP & 2003 & 2 & 10600.86 & 3.25E-05 & 2.781 & 85610.86 & Gamma \\
\hline FTR $>1 \mathrm{H}$ & MDP & 2004 & 0 & 8980.49 & 9.30E-06 & 0.781 & 83990.49 & Gamma \\
\hline $\mathrm{FTR}>1 \mathrm{H}$ & MDP & 2005 & 1 & 7681.39 & 2.15E-05 & 1.781 & 82691.39 & Gamma \\
\hline $\mathrm{FTR}>1 \mathrm{H}$ & MDP & 2006 & 0 & 7864.61 & $9.42 E-06$ & 0.781 & 82874.61 & Gamma \\
\hline $\mathrm{FTR}>1 \mathrm{H}$ & MDP & 2007 & 0 & 9252.11 & 9.27E-06 & 0.781 & 84262.11 & Gamma \\
\hline $\mathrm{FTR}>1 \mathrm{H}$ & MDP & 2008 & 0 & 6867.46 & 9.54E-06 & 0.781 & 81877.46 & Gamma \\
\hline $\mathrm{FTR}>1 \mathrm{H}$ & MDP & 2009 & 0 & 7435.46 & 9.47E-06 & 0.781 & 82445.46 & Gamma \\
\hline $\mathrm{FTR}>1 \mathrm{H}$ & MDP & 2010 & 0 & 8978.86 & 9.30E-06 & 0.781 & 83988.86 & Gamma \\
\hline $\mathrm{FTR}>1 \mathrm{H}$ & MDP & 2011 & 0 & 8896.77 & 9.31E-06 & 0.781 & 83906.77 & Gamma \\
\hline FTR $>1 \mathrm{H}$ & MDP & 2012 & 0 & 8136.92 & 9.39E-06 & 0.781 & 83146.92 & Gamma \\
\hline FTR>1H & MDP & 2013 & 1 & 8807.85 & 2.12E-05 & 1.781 & 83817.85 & Gamma \\
\hline $\mathrm{FTR}>1 \mathrm{H}$ & MDP & 2014 & 2 & 7880.31 & 3.36E-05 & 2.781 & 82890.31 & Gamma \\
\hline FTR $>1 \mathrm{H}$ & TDP & 1998 & 2 & 328.46 & $1.74 \mathrm{E}-03$ & 14.5 & 8356.2 & Gamma \\
\hline $\mathrm{FTR}>1 \mathrm{H}$ & TDP & 1999 & 0 & 2471.97 & 1.19E-03 & 12.5 & 10499.71 & Gamma \\
\hline $\mathrm{FTR}>1 \mathrm{H}$ & TDP & 2000 & 0 & 524.78 & 1.46E-03 & 12.5 & 8552.52 & Gamma \\
\hline $\mathrm{FTR}>1 \mathrm{H}$ & TDP & 2001 & 1 & 480.26 & 1.59E-03 & 13.5 & 8508 & Gamma \\
\hline FTR $>1 \mathrm{H}$ & TDP & 2002 & 0 & 1161.27 & 1.36E-03 & 12.5 & 9189.01 & Gamma \\
\hline $\mathrm{FTR}>1 \mathrm{H}$ & TDP & 2003 & 0 & 1394.97 & 1.33E-03 & 12.5 & 9422.71 & Gamma \\
\hline FTR>1H & TDP & 2004 & 3 & 299.85 & 1.86E-03 & 15.5 & 8327.59 & Gamma \\
\hline $\mathrm{FTR}>1 \mathrm{H}$ & TDP & 2005 & 1 & 214.92 & 1.64E-03 & 13.5 & 8242.66 & Gamma \\
\hline $\mathrm{FTR}>1 \mathrm{H}$ & TDP & 2006 & 2 & 186.97 & 1.77E-03 & 14.5 & 8214.71 & Gamma \\
\hline $\mathrm{FTR}>1 \mathrm{H}$ & TDP & 2007 & 0 & 205.35 & $1.52 \mathrm{E}-03$ & 12.5 & 8233.09 & Gamma \\
\hline FTR>1H & TDP & 2008 & 1 & 222.2 & 1.64E-03 & 13.5 & 8249.94 & Gamma \\
\hline
\end{tabular}


Table 6. (continued).

\begin{tabular}{|c|c|c|c|c|c|c|c|c|}
\hline \multirow{2}{*}{$\begin{array}{c}\text { Failure } \\
\text { Mode }\end{array}$} & \multirow[b]{2}{*}{ Component } & \multirow[b]{2}{*}{ Year } & \multirow{2}{*}{$\begin{array}{l}\text { Number of } \\
\text { Failures }\end{array}$} & \multirow{2}{*}{$\begin{array}{l}\text { Demands/ } \\
\text { Run Hours }\end{array}$} & \multicolumn{4}{|c|}{ Bayesian Update } \\
\hline & & & & & Mean & Post A & Post B & Distribution \\
\hline $\mathrm{FTR}>1 \mathrm{H}$ & TDP & 2009 & 0 & 202.03 & $1.52 \mathrm{E}-03$ & 12.5 & 8229.77 & Gamma \\
\hline $\mathrm{FTR}>1 \mathrm{H}$ & TDP & 2010 & 0 & 216.05 & $1.52 \mathrm{E}-03$ & 12.5 & 8243.79 & Gamma \\
\hline $\mathrm{FTR}>1 \mathrm{H}$ & TDP & 2011 & 2 & 352.65 & 1.73E-03 & 14.5 & 8380.39 & Gamma \\
\hline FTR>1H & TDP & 2012 & 3 & 194.53 & $1.89 \mathrm{E}-03$ & 15.5 & 8222.27 & Gamma \\
\hline $\mathrm{FTR}>1 \mathrm{H}$ & TDP & 2013 & 1 & 230.58 & $1.63 \mathrm{E}-03$ & 13.5 & 8258.32 & Gamma \\
\hline FTR $>1 \mathrm{H}$ & TDP & 2014 & 2 & 208.23 & 1.76E-03 & 14.5 & 8235.97 & Gamma \\
\hline FTS & MDP & 1998 & 4 & 1685.12 & 1.59E-03 & 5.948 & 3735.12 & Beta \\
\hline FTS & MDP & 1999 & 5 & 1636.93 & $1.88 \mathrm{E}-03$ & 6.948 & 3685.93 & Beta \\
\hline FTS & MDP & 2000 & 3 & 1726.26 & $1.31 \mathrm{E}-03$ & 4.948 & 3777.26 & Beta \\
\hline FTS & MDP & 2001 & 3 & 1807.29 & $1.28 \mathrm{E}-03$ & 4.948 & 3858.29 & Beta \\
\hline FTS & MDP & 2002 & 0 & 1893.23 & 4.93E-04 & 1.948 & 3947.23 & Beta \\
\hline FTS & MDP & 2003 & 4 & 2035.1 & $1.45 E-03$ & 5.948 & 4085.1 & Beta \\
\hline FTS & MDP & 2004 & 0 & 2130.03 & 4.65E-04 & 1.948 & $4.18 E+03$ & Beta \\
\hline FTS & MDP & 2005 & 3 & 2204.32 & 1.16E-03 & 4.948 & $4.26 \mathrm{E}+03$ & Beta \\
\hline FTS & MDP & 2006 & 4 & 1938.64 & 1.49E-03 & 5.948 & $3.99 \mathrm{E}+03$ & Beta \\
\hline FTS & MDP & 2007 & 4 & 2220.11 & 1.39E-03 & 5.948 & $4.27 E+03$ & Beta \\
\hline FTS & MDP & 2008 & 0 & 2007.37 & 4.79E-04 & 1.948 & $4.06 \mathrm{E}+03$ & Beta \\
\hline FTS & MDP & 2009 & 1 & 1841.06 & 7.56E-04 & 2.948 & $3.89 \mathrm{E}+03$ & Beta \\
\hline FTS & MDP & 2010 & 1 & 2078.23 & 7.13E-04 & 2.948 & $4.13 E+03$ & Beta \\
\hline FTS & MDP & 2011 & 2 & 2050.25 & 9.61E-04 & 3.948 & $4.10 \mathrm{E}+03$ & Beta \\
\hline FTS & MDP & 2012 & 3 & 1860.84 & 1.26E-03 & 4.948 & $3.91 \mathrm{E}+03$ & Beta \\
\hline FTS & MDP & 2013 & 4 & 2055.01 & $1.45 \mathrm{E}-03$ & 5.948 & $4.11 \mathrm{E}+03$ & Beta \\
\hline FTS & MDP & 2014 & 2 & 1799.73 & 1.02E-03 & 3.948 & $3.85 E+03$ & Beta \\
\hline FTS & TDP & 1998 & 1 & 1065.11 & $1.60 \mathrm{E}-03$ & 1.9421 & $1.21 \mathrm{E}+03$ & Beta \\
\hline FTS & TDP & 1999 & 6 & 976.19 & 6.19E-03 & 6.9421 & $1.11 \mathrm{E}+03$ & Beta \\
\hline FTS & TDP & 2000 & 5 & 979.61 & $5.28 \mathrm{E}-03$ & 5.9421 & $1.12 \mathrm{E}+03$ & Beta \\
\hline FTS & TDP & 2001 & 3 & 913.85 & 3.72E-03 & 3.9421 & $1.05 E+03$ & Beta \\
\hline FTS & TDP & 2002 & 2 & 911.77 & 2.78E-03 & 2.9421 & $1.05 E+03$ & Beta \\
\hline FTS & TDP & 2003 & 5 & 974.78 & 5.31E-03 & 5.9421 & 1113.88 & Beta \\
\hline FTS & TDP & 2004 & 4 & 994.49 & 4.34E-03 & 4.9421 & 1134.59 & Beta \\
\hline FTS & TDP & 2005 & 4 & 994.88 & 4.34E-03 & 4.9421 & 1134.98 & Beta \\
\hline FTS & TDP & 2006 & 3 & 986.51 & 3.48E-03 & 3.9421 & 1127.61 & Beta \\
\hline FTS & TDP & 2007 & 4 & 971.91 & 4.42E-03 & 4.9421 & 1112.01 & Beta \\
\hline FTS & TDP & 2008 & 3 & 984.15 & $3.49 \mathrm{E}-03$ & 3.9421 & 1125.25 & Beta \\
\hline FTS & TDP & 2009 & 10 & 1089.29 & 8.86E-03 & 10.9421 & 1223.39 & Beta \\
\hline FTS & TDP & 2010 & 4 & 1147.46 & 3.82E-03 & 4.9421 & 1287.56 & Beta \\
\hline FTS & TDP & 2011 & 4 & 1122.68 & 3.90E-03 & 4.9421 & 1262.78 & Beta \\
\hline FTS & TDP & 2012 & 2 & 1047.37 & 2.47E-03 & 2.9421 & 1189.47 & Beta \\
\hline FTS & TDP & 2013 & 1 & 1129.13 & $1.52 \mathrm{E}-03$ & 1.9421 & 1272.23 & Beta \\
\hline FTS & TDP & 2014 & 12 & 1056.95 & $1.08 \mathrm{E}-02$ & 12.9421 & 1189.05 & Beta \\
\hline
\end{tabular}


Table 7. Basic event UA trending data.

\begin{tabular}{|c|c|c|c|c|c|c|c|c|}
\hline \multirow{2}{*}{$\begin{array}{l}\text { Failure } \\
\text { Mode }\end{array}$} & \multirow[b]{2}{*}{ Component } & \multirow[b]{2}{*}{ Year } & \multirow{2}{*}{$\begin{array}{l}\text { UA } \\
\text { Hours }\end{array}$} & \multirow{2}{*}{$\begin{array}{c}\text { Critical } \\
\text { Hours }\end{array}$} & \multicolumn{4}{|c|}{ Bayesian Update } \\
\hline & & & & & Mean & Post A & Post B & Distribution \\
\hline UA & MDP & 1998 & 4180 & 655697 & 7.24E-03 & 0.594 & 81.5 & Beta \\
\hline UA & MDP & 1999 & 4996 & 934480 & 5.15E-03 & 1.883 & 363.5 & Beta \\
\hline UA & MDP & 2000 & 5146 & 963225 & 4.87E-03 & 1.315 & 268.4 & Beta \\
\hline UA & MDP & 2001 & 4224 & 962348 & 4.39E-03 & 2.442 & 553.8 & Beta \\
\hline UA & MDP & 2002 & 3818 & 988117 & 3.71E-03 & 2.621 & 703.7 & Beta \\
\hline UA & MDP & 2003 & 4329 & 966360 & 4.03E-03 & 1.501 & 370.7 & Beta \\
\hline UA & MDP & 2004 & 3885 & 990896 & 3.64E-03 & 2.315 & 633.9 & Beta \\
\hline UA & MDP & 2005 & 3851 & 981394 & 3.68E-03 & 1.925 & 521.6 & Beta \\
\hline UA & MDP & 2006 & 3495 & 993315 & 3.11E-03 & 1.287 & 412.6 & Beta \\
\hline UA & MDP & 2007 & 3415 & 991570 & 3.31E-03 & 1.992 & 599.1 & Beta \\
\hline UA & MDP & 2008 & 3667 & 988561 & 3.32E-03 & 1.218 & 365.9 & Beta \\
\hline UA & MDP & 2009 & 2898 & 994989 & 2.61E-03 & 1.511 & 576.3 & Beta \\
\hline UA & MDP & 2010 & 3144 & 976748 & 3.09E-03 & 1.782 & 574.3 & Beta \\
\hline UA & MDP & 2011 & 3428 & 966489 & 3.43E-03 & 1.541 & 447.9 & Beta \\
\hline UA & MDP & 2012 & 3183 & 926068 & 3.10E-03 & 1.078 & 346.5 & Beta \\
\hline UA & MDP & 2013 & 3065 & 906883 & 3.10E-03 & 1.164 & 373.8 & Beta \\
\hline UA & MDP & 2014 & 3043 & 904947 & 3.05E-03 & 1.452 & 474.0 & Beta \\
\hline UA & TDP & 1998 & 3025 & 350430 & 8.72E-03 & 0.941 & 107.0 & Beta \\
\hline UA & TDP & 1999 & 2699 & 503558 & 5.42E-03 & 1.366 & 250.7 & Beta \\
\hline UA & TDP & 2000 & 2766 & 516118 & 5.33E-03 & 1.743 & 325.0 & Beta \\
\hline UA & TDP & 2001 & 3081 & 514966 & $6.14 \mathrm{E}-03$ & 1.153 & 186.6 & Beta \\
\hline UA & TDP & 2002 & 2423 & 517926 & 4.70E-03 & 2.019 & 427.8 & Beta \\
\hline UA & TDP & 2003 & 3029 & 505485 & 6.01E-03 & 1.434 & 237.1 & Beta \\
\hline UA & TDP & 2004 & 2993 & 521680 & 5.95E-03 & 1.486 & 248.2 & Beta \\
\hline UA & TDP & 2005 & 2928 & 523076 & 5.68E-03 & 2.968 & 519.4 & Beta \\
\hline UA & TDP & 2006 & 2832 & 525399 & 5.35E-03 & 1.226 & 228.0 & Beta \\
\hline UA & TDP & 2007 & 2290 & 529216 & 4.35E-03 & 1.041 & 238.5 & Beta \\
\hline UA & TDP & 2008 & 2413 & 526129 & 4.59E-03 & 1.402 & 304.1 & Beta \\
\hline UA & TDP & 2009 & 2704 & 530917 & 5.09E-03 & 0.990 & 193.5 & Beta \\
\hline UA & TDP & 2010 & 3222 & 508310 & $6.48 \mathrm{E}-03$ & 1.291 & 198.0 & Beta \\
\hline UA & TDP & 2011 & 2790 & 512711 & 5.52E-03 & 0.839 & 151.2 & Beta \\
\hline UA & TDP & 2012 & 2314 & 495453 & 4.66E-03 & 0.889 & 190.0 & Beta \\
\hline UA & TDP & 2013 & 2204 & 489741 & 4.51E-03 & 1.241 & 273.7 & Beta \\
\hline UA & TDP & 2014 & 2764 & 490066 & 5.67E-03 & 0.430 & 75.4 & Beta \\
\hline
\end{tabular}


Table 8 . Failure mode acronyms.

\begin{tabular}{ll}
\hline \multicolumn{1}{c}{ Failure Mode } & \multicolumn{1}{c}{ Failure Mode Description } \\
\hline FTLR & Fail to load/run \\
FTOC & Fail to open/close \\
FTOP & Fail to operate \\
FTR & Fail to run \\
FTR $<1$ H & Fail to run less than one hour (after start) \\
FTS & Fail to start \\
SO & Spurious operation \\
UA & Unavailability (maintenance or state of another component) \\
\hline
\end{tabular}


System Study

Auxiliary Feedwater
2014 Update December 2015 


\section{SYSTEM DESCRIPTION}

The main purpose of the AFW system is to provide feedwater to the steam generators to maintain a heat sink in the event of (1) a loss of main feedwater, (2) a reactor trip and loss of offsite power, and (3) a small break loss of coolant accident. The system, at some plants, can also provide a source of feedwater to the steam generators during plant startup and shutdown. However, the system cannot supply sufficient feedwater flow during power operation. At most plants, the system can only supply adequate feedwater to the steam generators with steam loads less than $5 \%$ of rated flow.

The safety-related function of the AFW system is to maintain water inventory in the steam generators for reactor residual heat removal when the main feedwater system is unavailable. The system is designed to automatically start and supply sufficient feedwater to prevent the relief of primary coolant through the pressurizer safety valves. The AFW system, in conjunction with the steam generators and the main steam line atmospheric relief and/or safety valves, is used to cool the reactor coolant system to the residual heat removal cut-in temperature. At this temperature, the residual heat removal system is used to further cool the reactor coolant system. The AFW system may also be used to temporarily hold the plant in a hot standby condition while main feedwater flow is being restored, with the option of cooling the reactor coolant system to the residual heat removal system initiation temperature.

The AFW system typically consists of at least two independent divisions. The divisions consist of a number of different combinations of electric-motor-driven and/or turbine-driven pump trains or diesel-driven pump trains. Electrical power, control, and instrumentation associated with each division are independent from one another. Typically, the electric-motor-driven pump trains make up one division and the turbine-driven pump train the other. Some plants have a diesel-driven pump in place of the turbine-driven pump, or a second turbine-driven pump in place of the electric-motor-driven pumps.

The AFW system is typically started automatically by the engineered safety features actuation system (ESFAS) or equivalent, depending on plant design and terminology. The ESFAS system automatic start signals include a predetermined low water level condition in one or more steam generators, a loss of the operating main feedwater pumps, a loss of electrical power on safety-related buses, and a safety injection signal. There are additional start signals, but these four are the most common. There is significant variation among the plants in how the system responds given a start signal. However, in most cases, a low-level condition in one steam generator starts only the electric-motor-driven pumps, while a low-level condition in two or more steam generators starts both the electric and turbine-driven pumps. For the plants that have two divisions consisting of one train per division (i.e., an electric-motor and turbine-driven pump train), most start signals start both pumps.

Feedwater flow to each steam generator is normally controlled by a flow control valve that will modulate either open or closed to maintain steam generator level. The flow control valve can be controlled either automatically or manually. A flow recirculation line is provided downstream of each pump discharge. The recirculation line allows for continuous flow back to the suction source to provide minimum flow protection for the pump. In addition, a test return line is provided downstream of each pump discharge to allow for either full or partial testing of the pumps. To limit the flow, as steam generator pressure lowers during a cool down, the system utilizes several different methods depending on plant design. Some plants use a current limiter that acts to increase downstream pump pressure thereby reducing motor amps, others use flow restricting orifices or pipe design configurations, and others use the flow control valve that modulates closed when a flow reduction signal is received.

The turbine for each turbine-driven pump is classified as an atmospheric discharge, non-condensing turbine. Typically, driving steam is supplied from the main steam lines upstream of the main steam isolation valves from at least two steam generators. (Design class 11 turbine steam supply is from one steam generator.) Each steam supply line to the turbine contains a normally closed fail-open air operated steam isolation valve. Some plants have a dc-powered motor-operated valve. A bypass is provided around each of these isolation valves with a flow- 
restricting orifice and a normally closed fail-to-open air-operated bypass isolation valve. The bypass provides a small, controlled rate of steam flow to the AFW turbine for warming the steam lines and turbine. Steam drain traps are provided in the low points of the steam line to drain condensate from the lines as condensate present in the steam lines could have an adverse effect on turbine reliability during an unplanned demand.

Each turbine is supplied with a hydraulic governor control valve, and a trip and throttle valve with motor reset capability. The turbine is brought up to speed by governor control upon being supplied with steam by opening the steam supply isolation valve(s). The governor then controls the turbine speed at the pump rated speed by modulating the governor control valve. The governor controlled turbine speed can be adjusted from the control room, the remote shutdown panel, or manually at the governor.

The turbine is stopped by remotely closing the trip throttle valve from the control room or the remote shutdown panel. The trip and throttle valve is automatically (electrically) tripped on turbine overspeed at $115 \%$ of rated speed. The electric overspeed trip can be reset from either the control room or remote shutdown panel. A mechanical overspeed trip also provides automatic overspeed protection at $125 \%$ of rated speed. The mechanical overspeed trip can only be reset at the trip and throttle valve.

Feedwater is supplied to both divisions through either a single condensate storage tank with separate suction supply lines or two storage tanks with redundant supply lines. Each tank typically will have its level maintained above the minimum volume needed to provide a net positive suction head to the pumps and allow for 6 hours of system operation. For extended operation of the system or as a backup for the storage tanks, an ensured source of water is provided from a service water system. The switchover to the ensured source can be accomplished by either an automatic re-alignment of the suction valves based on a sensed, low-suction pressure condition or manually by operator action depending on the plant design (typical alignment at most plants is by manual capability).

The AFW systems analyzed can be grouped into three different design classes based on the effective redundancy of the pumps. Each system typically consists of at least two independent divisions. The divisions consist of a number of motor-, turbine-, and/or diesel-driven pumps. In addition, some SPAR models include other sources of emergency feed water such as the startup feedwater pump(s). The configurations are shown in Table 9. 
Table 9. Listing of the AFW design classes.

\begin{tabular}{|c|c|c|c|c|c|}
\hline Class & Plant & $\begin{array}{l}\text { AFW } \\
\text { EDP }\end{array}$ & $\begin{array}{l}\text { AFW } \\
\text { MDP }\end{array}$ & $\begin{array}{l}\text { AFW } \\
\text { TDP }\end{array}$ & Other \\
\hline Class 2 & Arkansas 1 & & 1 & 1 & \\
\hline Class 2 & Braidwood 1 & 1 & 1 & & \\
\hline Class 2 & Braidwood 2 & 1 & 1 & & \\
\hline Class 2 & Byron 1 & 1 & 1 & & \\
\hline Class 2 & Byron 2 & 1 & 1 & & \\
\hline Class 2 & Crystal River 3 & 1 & & 1 & \\
\hline Class 2 & Prairie Island 1 & & 1 & 1 & $1^{a}$ \\
\hline Class 2 & Prairie Island 2 & & 1 & 1 & $1^{a}$ \\
\hline Class 2 & Seabrook & & 1 & 1 & $1^{\mathrm{b}}$ \\
\hline Class 3 & Arkansas 2 & & 1 & 1 & $1^{b}$ \\
\hline Class 3 & Beaver Valley 2 & & 2 & 1 & \\
\hline Class 3 & Callaway & & 2 & 1 & \\
\hline Class 3 & Catawba 1 & & 2 & 1 & \\
\hline Class 3 & Catawba 2 & & 2 & 1 & \\
\hline Class 3 & Comanche Peak 1 & & 2 & 1 & \\
\hline Class 3 & Comanche Peak 2 & & 2 & 1 & \\
\hline Class 3 & Cook 1 & & 2 & 1 & \\
\hline Class 3 & Cook 2 & & 2 & 1 & \\
\hline Class 3 & Diablo Canyon 1 & & 2 & 1 & \\
\hline Class 3 & Diablo Canyon 2 & & 2 & 1 & \\
\hline Class 3 & Farley 1 & & 2 & 1 & \\
\hline Class 3 & Farley 2 & & 2 & 1 & \\
\hline Class 3 & Fort Calhoun & 1 & 1 & 1 & \\
\hline Class 3 & Harris & & 2 & 1 & \\
\hline Class 3 & Indian Point 2 & & 2 & 1 & \\
\hline Class 3 & Indian Point 3 & & 2 & 1 & \\
\hline Class 3 & Kewaunee & & 2 & 1 & \\
\hline Class 3 & McGuire 1 & & 2 & 1 & \\
\hline Class 3 & McGuire 2 & & 2 & 1 & \\
\hline Class 3 & Millstone 2 & & 2 & 1 & \\
\hline Class 3 & Millstone 3 & & 2 & 1 & \\
\hline Class 3 & North Anna 1 & & 2 & 1 & \\
\hline Class 3 & North Anna 2 & & 2 & 1 & \\
\hline Class 3 & Oconee 1 & & 2 & 1 & \\
\hline Class 3 & Oconee 2 & & 2 & 1 & \\
\hline Class 3 & Oconee 3 & & 2 & 1 & \\
\hline
\end{tabular}

\begin{tabular}{|c|c|c|c|c|c|}
\hline Class & Plant & $\begin{array}{l}\text { AFW } \\
\text { EDP }\end{array}$ & $\begin{array}{l}\text { AFW } \\
\text { MDP }\end{array}$ & $\begin{array}{l}\text { AFW } \\
\text { TDP }\end{array}$ & Other \\
\hline Class 3 & Palisades & & 2 & 1 & \\
\hline Class 3 & Palo Verde 1 & & 2 & 1 & \\
\hline Class 3 & Palo Verde 2 & & 2 & 1 & \\
\hline Class 3 & Palo Verde 3 & & 2 & 1 & \\
\hline Class 3 & Point Beach 1 & & 2 & 1 & \\
\hline Class 3 & Point Beach 2 & & 2 & 1 & \\
\hline Class 3 & Robinson 2 & & 2 & 1 & \\
\hline Class 3 & Salem 1 & & 2 & 1 & \\
\hline Class 3 & Salem 2 & & 2 & 1 & \\
\hline Class 3 & San Onofre 2 & & 2 & 1 & \\
\hline Class 3 & San Onofre 3 & & 2 & 1 & \\
\hline Class 3 & Sequoyah 1 & & 2 & 1 & \\
\hline Class 3 & Sequoyah 2 & & 2 & 1 & \\
\hline Class 3 & St. Lucie 1 & & 2 & 1 & \\
\hline Class 3 & St. Lucie 2 & & 2 & 1 & \\
\hline Class 3 & Summer & & 2 & 1 & \\
\hline Class 3 & Three Mile Island 1 & & 2 & 1 & \\
\hline Class 3 & Turkey Point 3 & & & 3 & \\
\hline Class 3 & Turkey Point 4 & & & 3 & \\
\hline Class 3 & Vogtle 1 & & 2 & 1 & \\
\hline Class 3 & Vogtle 2 & & 2 & 1 & \\
\hline Class 3 & Waterford 3 & & 2 & 1 & \\
\hline Class 3 & Watts Bar 1 & & 2 & 1 & \\
\hline Class 3 & Wolf Creek & & 2 & 1 & \\
\hline Class 4 & Beaver Valley 1 & & 2 & 1 & 1 \\
\hline Class 4 & Calvert Cliffs 1 & & 2 & 2 & \\
\hline Class 4 & Calvert Cliffs 2 & & 2 & 2 & \\
\hline Class 4 & Davis-Besse & & 1 & 2 & 1 \\
\hline Class 4 & Ginna & & 2 & 1 & 2 \\
\hline Class 4 & South Texas 1 & & 3 & 1 & \\
\hline Class 4 & South Texas 2 & & 3 & 1 & \\
\hline Class 4 & Surry 1 & & 2 & 1 & 3 \\
\hline Class 4 & Surry 2 & & 2 & 1 & 3 \\
\hline
\end{tabular}


System Study

Auxiliary Feedwater
2014 Update December 2015 


\section{REFERENCES}

1. Nuclear Regulatory Commission, Component Reliability Data Sheets Update 2010, January 2012, http://nrcoe.inl.gov/resultsdb/publicdocs/AvgPerf/ComponentReliabilityDataSheets2010.pdf

2. S.A. Eide et al., Industry-Average Performance for Components and Initiating Events at U.S. Commercial Nuclear Power Plants, Nuclear Regulatory Commission, NUREG/CR-6928, February 2007. 